\title{
Una nueva subespecie de Heraclides androgeus (Lepidoptera: Papilionidae) y sus aspectos biogeográficos
}

\author{
Isabel Vargas-Fernández, Armando Luis-Martínez \& Jorge Llorente-Bousquets* \\ Departamento de Biología Evolutiva, Facultad de Ciencias, Universidad Nacional Autónoma de México, México, D.F. \\ 04510; ivf@ciencias.unam.mx, alm@ciencias.unam.mx, jlb@hp.fciencias.unam.mx \\ * Correspondencia
}

Recibido 19-III-2012. C Corregido 23-VIII-2012. Aceptado 24-IX-2012.

\begin{abstract}
A new subspecies of Heraclides androgeus (Lepidoptera: Papilionidae) and its biogeographical aspects. Heraclides androgeus epidaurus was described and illustrated by Godman \& Salvin in 1890 based on specimens obtained in Veracruz, indicating that their distribution encompassed both the Pacific and Atlantic sides of Mexico. Later authors commented that there were morphological differences between the male wings from both populations. We analyzed, described and nominated Heraclides androgeus reyesorum ssp. nov. Vargas, Llorente \& Luis distributed in the Mexican Pacific coast, based on 62 specimens, and compared it with H. a. epidaurus from the Gulf of Mexico, based on more than 200 specimens housed at UNAM: Museo de Zoología, Facultad de Ciencias and the Colección Nacional de Insectos of the Instituto de Biología, as well as some collections from the USA. The main characters were the width of the yellow and black bands on forewings in males, which had a significant difference between the populations of both sides of Mexico, although some characters were variable and showed partial overlap. In the hindwings, the differences were the extent of the subterminal lunules in dorsal and ventral view. We also analyzed the male genitalia, finding notorious differences in both sclerotic processes of the harpe. Subspecific differences between females refer to the brightness and extent of green spots on the hindwings and the extent of lunules in the ventral view. The greatest abundance of H. a. reyesorum ssp. nov. was in the tropical deciduous forest, with gallery forest and in the lower range of the cloud forest, present at altitudes of 500-800m and $1000-1750 \mathrm{~m}$, respectively. We discussed the pattern of endemism due to historical vicariant processes and explain the presence of the new subspecies of $\mathrm{H}$. androgeus and other taxa of specific level. Rev. Biol. Trop. 61 (2): 711-733. Epub 2013 June 01.
\end{abstract}

Key words: Papilionidae, endemism, vicariance, distribution, Mexican Pacific Coast, semi-evergreen seasonal forest, genitalia.

El género Heraclides Hübner, [1819] se compone de 28 especies en el Neotrópico (Lamas 2004). El mismo autor reconoce tres subespecies para Heraclides androgeus (Cramer, 1775); de ellas, Heraclides androgeus epidaurus (Godman \& Salvin, 1890) es la más septentrional. Originalmente fue descrita bajo el género Papilio por Godman \& Salvin, como especie cercana y distinta de androgeus, con base en ejemplares de México (Durango y varios lugares de Veracruz) y Centroamérica: Guatemala, Nicaragua, Costa Rica y Panamá. Según estos autores, las hembras de México y Panamá difieren muy poco entre sí y mencionan que allí prevalece solo una forma femenina; además, enfatizan que las figuras de Cramer y la opinión de Bates parecen indicar dos formas del Valle del Amazonas y Guiana. Godman \& Salvin admiten que su Papilio epidaurus se distribuye en las sierras de ambas vertientes de México y Guatemala, así también que las hembras resultan escasas y difíciles de encontrar.

Rothschild \& Jordan (1906) presentan una sinonimia bastante completa y con ello amplían su distribución (Cuba, Haití, Puerto Rico, Santa Lucía y Honduras). Ellos anotan que las áreas amarillas en las Alas Anteriores (AA) y Alas Posteriores (AP) de ejemplares machos, de lo 
que consideran Papilio androgeus epidaurus, son más amplias en comparación con las formas sudamericanas, y que la mancha amarilla situada al frente de la bifurcación subcostal de las AA también es de mayor tamaño. Los mismos autores mantienen y destacan que solo se conoce una forma femenina y advierten que la mancha de color azul verdoso en el área discal de las AP es más extensa que en las otras formas, entre otros caracteres. Ellos mismos agregan a la distribución en México, áreas de Veracruz (Orizaba y Zongolica).

Los límites geográficos entre las subespecies epidaurus y androgeus los trazan LeCrom et al. (2004); éstos ocurren en Colombia. H. $a$. androgeus se circunscribe al sur de los Andes en las tierras bajas de la cuenca amazónica y la Orinoquia. H. a. epidaurus en las cuencas de los ríos Magdalena, Medellín y Atrato de Colombia hasta la costa Atlántica de ese país.

Carlos Christian Hoffmann (1940), en su Catálogo de los Lepidópteros Mexicanos, reconoce a $H$. androgeus epidaurus con la siguiente distribución en México: "Región oriental hasta el sur de Tamaulipas, Sierra Madre Occidental hasta Sinaloa y Durango". Esto es por las dos vertientes en áreas cálidas y templado-cálidas en la terminología humboldtiana.

Casi medio siglo después, Beutelspacher $\&$ Howe (1984) advierten que en esta subespecie, el margen negro de las AP de machos del Pacífico mexicano muestra tendencia a acercarse al extremo de la célula discal, y aunque piensan en una subespecie distinta lo consideran variabilidad poblacional, pues incluyen algunos ejemplares de la vertiente del Golfo con aparentes tendencias similares. Por referencias y el material consultado, incrementaron la distribución de áreas de ocurrencia de epidaurus en México, lo que grafican en un mapa.

En efecto, la aparente tendencia en la reducción de la banda amarilla de las AA en esas poblaciones ocurre, pero tales ejemplares nunca alcanzan la condición de los ejemplares en las poblaciones de la vertiente del Pacífico. Por otra parte, al examinar y comparar otra decena de caracteres en machos y hembras del verdadero epidaurus y de la nueva subespecie, nos permiten distinguir, describir y denominar a las poblaciones del Pacífico mexicano.

Los genitales masculinos de epidaurus de México fueron ilustrados por primera vez por Godman \& Salvin (1890) y casi un siglo después por Beutelspacher \& Howe (1984). En ambas publicaciones no se destacan diferencias específicas y los ejemplares -al parecer- solo provienen de la vertiente del Golfo, posiblemente Veracruz.

H. a. epidaurus también se considera por Tyler et al. (1994), quienes ilustran gruesamente los genitales masculinos, ejemplares adultos de machos y hembras, dorsal y ventralmente, así como el mapa de distribución general, entre los principales aspectos.

El propósito del presente estudio es la descripción de $H$. androgeus del Pacífico mexicano. A lo largo de más de 30 años, hemos estudiado este taxón y reunido evidencia de que está formado por dos subespecies, cada una con caracteres propios que las distinguen. Vargas et al. (1996) y Warren et al. (1998) incluyeron a $H$. androgeus dentro del género Calaides, e indicaron que la subespecie localizada en Jalisco y Colima era nueva.

\section{MATERIALES Y MÉTODOS}

La descripción del nuevo taxón se basó en el análisis de 62 especímenes del Museo de Zoología de la Facultad de Ciencias de la UNAM (46 machos y 16 hembras), provenientes de diversos estudios faunísticos efectuados a lo largo de 33 años. Los sitios de colecta en orden geográfico y cronológico son: Sierra de San Juan, Nayarit (Warren \& Llorente 1999, Llorente et al. 2004), Sierra de Manantlán, Jalisco-Colima (Vargas et al. 1996, 1999, Warren et al. 1998); otros estudios los tenemos en curso en los estados de Michoacán y Oaxaca, vertiente del Pacífico. Adicionalmente, se tuvo acceso a los datos distribucionales del Instituto de Biología (30 registros de los estados de Colima, Guerrero, Jalisco y Michoacán) y de las siguientes colecciones: Museo de Historia Natural de San Diego, CA (SDNHM), National Museum of Natural History, Smithsonian 
Institution, Washington, DC (USNM) y de la Colección del Museo Allyn (AME), hoy en el McGuire Center for Lepidoptera and Biodiversity, Gainesville, FL (MGCL), que solo fueron utilizados para los gráficos de fenología y de distribución, esto es, no se incluyeron dentro de la serie típica.

De $H$. a. epidaurus se contó con la información de más de 200 ejemplares (Apéndice 1); en su mayor parte de las dos colecciones alojadas en la UNAM: El Museo de Zoología de la Facultad de Ciencias (MZFC: 95 registros: 71 machos, 24 hembras) y la Colección Nacional de Insectos del Instituto de Biología (CNIN: 60 registros). De otras colecciones de los Estados Unidos (54 registros de seis colecciones); además de las ya mencionadas AME (hoy MGCL), USNM y SDNHM, están: AMNH=American Museum of Natural History; $\mathrm{CAS}=$ California Academy of Sciences; LACM=Natural History Museum of Los Angeles County. Los 95 ejemplares de H. a. epidaurus del MZFC se tuvieron disponibles para su análisis y comparación con los 62 individuos de la nueva subespecie de Heraclides androgeus.
Los caracteres utilizados y valorados en los conjuntos poblacionales de $H$. androgeus se describen en el cuadro $1 \mathrm{y}$ se ilustran en la figura 1. El cuadro 2 muestra una comparación de los caracteres entre las dos subespecies.

Para la nomenclatura de las áreas alares se ha seguido a Howe (1975) y Brown \& Faulkner (1984); para la precisión del color en las descripciones a menudo añadimos, entre paréntesis, las claves que corresponden en el Atlas de colores de Klüppers (1996).

Con el propósito de comparar cuantitativamente algunos caracteres de ejemplares de la vertiente Pacífica vs. la vertiente del Golfo, se estudiaron los 46 machos disponibles de $H$. androgeus ssp. nov. vs. los 71 de $H$. androgeus epidaurus, tomando las medidas en mm de cuatro de los caracteres alares de las AA (Apéndice 2), ilustrados en la figura $1 \mathrm{y}$ descritos en el cuadro 1 , además del Carácter $3 / 4$ que se trata del cociente entre el carácter 3 y el carácter 4. Las medidas se realizaron con un Vernier (Calibrador) DIGIMATIC Mitutoyo, Manual No. 2071M, Serie No. 500. Se eligió a estos caracteres porque a simple vista destacan

CUADRO 1

Descripción de los caracteres alares cuantitativos y cualitativos

TABLE 1

Description of quantitative and qualitative wing characters

\begin{tabular}{|c|c|}
\hline Carácter & Descripción del carácter \\
\hline 1 & Envergadura Alar (AA)--línea recta comprendida de la base al ápice \\
\hline 2 & Banda amarilla del margen posterior (AA)--línea recta medida sobre el margen posterior, banda amarilla \\
\hline 3 & $\begin{array}{l}\text { Banda amarilla de la vena } \mathrm{CuA}_{1}(\mathrm{AA}) \text {--línea recta medida sobre } \mathrm{CuA}_{1} \text {, desde la célula discal }(\mathrm{CD}) \text { hasta el } \\
\text { inicio de la banda negra }\end{array}$ \\
\hline 4 & Banda negra de la vena $\mathrm{CuA}_{1}(\mathrm{AA})-$-amplitud de la banda negra medida en línea recta sobre $\mathrm{CuA}_{1}$ \\
\hline $3 / 4$ & Cociente de caracteres 3 y 4 (AA) \\
\hline 5 & Tamaño y anchura de las cinco lúnulas amarillas subterminales (AP) \\
\hline 6 & Amplitud de la banda subterminal negra y su cercanía de la CD (AP) \\
\hline 7 & Tamaño de la mancha naranja situada en el tornus (AP) \\
\hline 8 & Envergadura alar (AA)--línea recta comprendida de la base al ápice \\
\hline 9 & Color y brillo por regiones alares (AA) \\
\hline 10 & Color y brillo por regiones alares (AP) \\
\hline 11 & Posición de manchas verde claro con respecto a la CD (AP) \\
\hline 12 & Anchura de las lúnulas amarillas subterminales (AP) \\
\hline
\end{tabular}

Los caracteres $1-4$ y $3 / 4$ son cuantitativos.

Los caracteres 1 a 7 corresponden a los machos y 8 a 12 a las hembras; todos los caracteres corresponden a la vista dorsal, excepto el 12 , que es ventral. 
CUADRO 2

Comparación entre H. a. epidaurus y H. a. reyesorum ssp. nov. (medidas promedio)

TABLE 2

Comparison of $H$. a. epidaurus and $H$. a. reyesorum ssp. nov. (measured average)

\begin{tabular}{|c|c|c|}
\hline \multicolumn{3}{|c|}{ Machos } \\
\hline Carácter & H. androgeus epidaurus & H. a. reyesorum ssp. nov. \\
\hline Envergadura (Carácter 1) & $\begin{array}{l}\text { De angosta, mediana o amplia } \\
(60.2 \mathrm{~mm})\end{array}$ & $\begin{array}{l}\text { Generalmente de medianas a grandes } \\
(62.1 \mathrm{~mm})\end{array}$ \\
\hline $\begin{array}{l}\text { Amplitud de la banda amarilla del } \\
\text { margen posterior (Carácter 2) }\end{array}$ & $\begin{array}{l}\text { De angosta, mediana o amplia } \\
(20.3 \mathrm{~mm})\end{array}$ & $\begin{array}{l}\text { La gran mayoría de angosta a } \\
\text { mediana }(18.9 \mathrm{~mm})\end{array}$ \\
\hline $\begin{array}{l}\text { Amplitud de la banda amarilla, medida } \\
\left.\text { sobre la vena } \mathrm{CuA}_{1} \text { (Carácter } 3\right)\end{array}$ & $\begin{array}{l}\text { De angosta, mediana o amplia } \\
(13.7 \mathrm{~mm})\end{array}$ & $\begin{array}{l}\text { La gran mayoría de angosta a } \\
\text { mediana }(13.0 \mathrm{~mm})\end{array}$ \\
\hline $\begin{array}{l}\text { Amplitud de la banda negra, medida } \\
\left.\text { sobre la vena } \mathrm{CuA}_{1} \text { (Carácter } 4\right)\end{array}$ & De angosta a mediana $(5.9 \mathrm{~mm})$ & De mediana a grande $(7.3 \mathrm{~mm})$ \\
\hline $\begin{array}{l}\text { Cociente Carácter 3/Carácter } 4 \text { (Carácter } \\
3 / 4 \text { ) }\end{array}$ & De mediano a grande $(2.3 \mathrm{~mm})$ & De pequeño a mediano (1.8mm) \\
\hline $\begin{array}{l}\text { Amplitud de banda negra respecto a la } \\
\text { amarilla }\end{array}$ & $1 / 3$ a $3 / 5$ de la banda amarilla & $1 / 2$ a $2 / 3$ de la banda amarilla \\
\hline $\begin{array}{l}\text { Amplitud media de lúnulas amarillas } \\
\text { subterminales en AP, vista dorsal }\end{array}$ & Angostas la gran mayoría & $\begin{array}{l}\text { La mayoría amplias o medianamente } \\
\text { amplias }\end{array}$ \\
\hline $\begin{array}{l}\text { Venas discocelulares } \mathrm{M}_{2}-\mathrm{M}_{3}(\mathrm{dci}) \text { y } \mathrm{M}_{1} \\
\text { postdiscal en AP }\end{array}$ & La gran mayoría color amarillo & $\begin{array}{l}\text { A menudo son negras, en especial } \\
\text { cuando la banda obscura postdiscal } \\
\text { toca la } \mathrm{CD}\end{array}$ \\
\hline Abdomen & $\begin{array}{l}\text { Banda negra pleural angosta }(1 \mathrm{~mm}) \text {, } \\
\text { tendiendo a desaparecer }\end{array}$ & $\begin{array}{l}\text { Banda negra pleural de angosta a } \\
\text { amplia }(1-2.5 \mathrm{~mm})\end{array}$ \\
\hline \multicolumn{3}{|c|}{ Genitales } \\
\hline Proceso dorsal esclerosado del harpagón & $\begin{array}{l}\text { Alargado y amplio en su base, hasta } \\
\text { cuatro veces la altura; el margen } \\
\text { dentado homogéneo }\end{array}$ & $\begin{array}{l}\text { Subtriangular, dimensión de la altura } \\
\text { similar a la base; margen dentado } \\
\text { heterogéneo }\end{array}$ \\
\hline Proceso ventral esclerosado del harpagón & En forma de espina, uniforme y recto & $\begin{array}{l}\text { Se presenta como una espina más } \\
\text { gruesa muy pronunciada y angular en } \\
\text { el tercio más distal, abombada en su } \\
\text { primer tercio }\end{array}$ \\
\hline
\end{tabular}

\begin{tabular}{|c|c|c|}
\hline \multicolumn{3}{|c|}{ Hembras } \\
\hline Envergadura promedio & $\begin{array}{l}\text { Generalmente medianas } \\
(61.8 \mathrm{~mm})\end{array}$ & $\begin{array}{l}\text { Generalmente de medianas a grandes } \\
(63.3 \mathrm{~mm})\end{array}$ \\
\hline Forma de mancha verde RS-M $\mathrm{M}_{1}$ en AP & $\begin{array}{l}\text { Se extiende hacia el área basal más } \\
\text { allá de la } C D \text {, con un borde difuso }\end{array}$ & $\begin{array}{l}\text { Borde redondeado hacia el área basal; } \\
\text { su extensión alineada al límite de la } \\
\text { discocelular } \mathrm{M}_{1}-\mathrm{M}_{2}\end{array}$ \\
\hline Color de fondo en AA y región basal & Negro verdoso con brillo & Negro verdoso mate \\
\hline
\end{tabular}
en AP

Mancha en área apical de CD color verde

Siempre se presenta, a veces es

Rara vez se presenta

Amplitud de lúnulas amarillas subterminales en AP, vista ventral amplia

Angostas en su mayoría A menudo medianamente amplias a muy amplias

\section{Distribución}

Distribución geográfica

En la vertiente costera del Atlántico, desde Chiapas hasta Tamaulipas. Entre el nivel del mar y $1750 \mathrm{~m}$

Provincia biogeográfica Provincia Golfo de México (Morrone, 2001) 


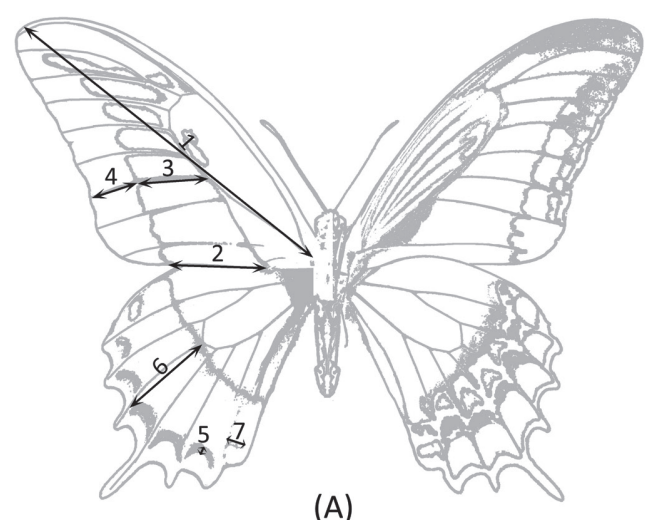

(A)

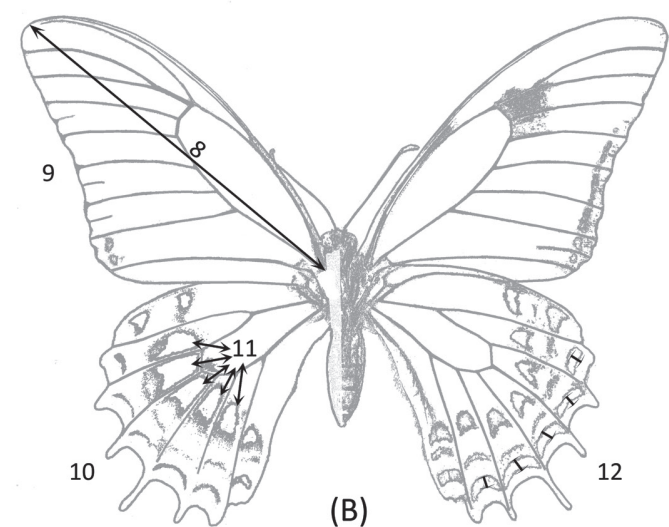

(B)

Fig. 1. Caracteres alares (A) macho; (B) hembra.Vista dorsal (izquierda) y ventral (derecha). Los números corresponden a los caracteres: 1-Envergadura Alar; 2-Banda amarilla del margen posterior; 3-Banda amarilla de la vena CuA 1 ; 4-Banda negra de la vena $\mathrm{CuA}_{1}$; 5-Tamaño y anchura de las cinco lúnulas amarillas subterminales; 6-Amplitud de la banda subterminal negra y su cercanía de la Célula discal o CD; 7-Tamaño de la mancha naranja situada en el tornus; 8-Envergadura alar; 9,10-Color y brillo por regiones alares; 11-Posición de manchas verde claro con respecto a la CD; 12-Anchura de las lúnulas amarillas subterminales.

Fig. 1. Wing characters (A) male; (B) female. Dorsal view (left) and ventral (right). Numbers correspond to characters: 1-Wing Span; 2-Yellow band of posterior margin; 3-Yellow band of vein $\mathrm{CuA}_{1}$; 4-Black band of vein $\mathrm{CuA}$; 5-Size and width of five yellow subterminal lunules; 6-Extent of black subterminal band and its proximity to Discal cell or CD; 7-Size of orange spot located at tornus; 8-Wing Span; 9, 10-Color and brightness of wing regions; 11-Light green spots position with respect to $\mathrm{CD} ; 12$-Width of yellow subterminal lunules.

diferencias entre las dos subespecies respecto a la amplitud de las bandas amarilla postmediana y negra subterminal en las AA. La medida de la banda amarilla se tomó a dos niveles del ala (sobre el margen posterior y sobre la vena $\mathrm{CuA}_{1}$ ) debido a que la banda se estrecha hacia la zona subapical.

Se aplicó a estos caracteres la prueba no paramétrica U de Mann-Whitney, comparándolos en las dos subespecies, tratándose como muestras independientes.

Se diseccionaron en sus genitales el holotipo y dos paratipos machos. Los genitales fueron removidos al desprender el abdomen con unas pinzas y se maceró en $\mathrm{KOH}$ al $10 \%$, colocándolo posteriormente en un godete de 24 a 48 horas. Después, el abdomen se lavó para remover la potasa y se colocó en una caja de petri pequeña con alcohol al $70 \%$, para efectuar la disección al microscopio con pinzas de punta fina, quitando primero las escamas de las valvas y luego el exceso de tejidos blandos poco a poco, hasta llegar al área genital y dejar completamente libre la armadura, extrayendo el edeago. Finalmente se aclaró con xilol durante unas horas. Se prefirió estudiarlos sin montar para observar las estructuras apreciando su volumen desde distintos ángulos. Finalmente se guardaron en un microtubo con glicerina.

\section{RESULTADOS}

\section{Heraclides androgeus reyesorum ssp. nov. Vargas, Llorente \& Luis}

\section{English diagnosis of new taxon}

It can be distinguished from $H$. a. epidaurus by the following characters: Male. FW, size $62.1 \mathrm{~mm}$, black band wider in dorsal view. HW, yellow subterminal lunules of greater amplitude, discocellulars often black. Black pleural abdominal band broader. Genitalia. Harpe with subtriangular sclerotized dorsal process and ventral process angular, like a thick thorn in the distal third.

Female. FW, size $63.3 \mathrm{~mm}$, color dull greenish black background. HW green spot 
in $\mathrm{Rs}-\mathrm{M}_{1}$ rounded and well-defined edge to the base, limited to the $\mathrm{M}_{1}-\mathrm{M}_{2}$ discocellular; yellow subterminal lunules very broad in ventral view.

\section{Descripción}

Macho (Fig. 2)

Envergadura AA $=62.19 \mathrm{~mm}$

$(51.28-68.23, \mathrm{n}=46)$

Antenas negras; palpos densamente pubescentes, la mayor parte amarillos y en el borde interno negros. La frente es negra con una línea amarilla a cada lado. El tórax es negro en el dorso, con algunas escamas amarillas en el borde interior de las tégulas; ventralmente es amarillo. El abdomen es amarillo con una banda dorsal negra a lo largo de éste, que a menudo alcanza de 2 a $2.5 \mathrm{~mm}$ de amplitud.

Alas anteriores, vista dorsal: Negras, con una banda diagonal amarilla $\left(\mathrm{N}_{00} \mathrm{~A}_{60-80}\right.$ $\mathrm{M}_{20}$ ) en el área postmediana, desde la zona apical en $\mathrm{R}_{3}$ hasta el margen posterior, por debajo de la CD. Esta banda consiste de ocho

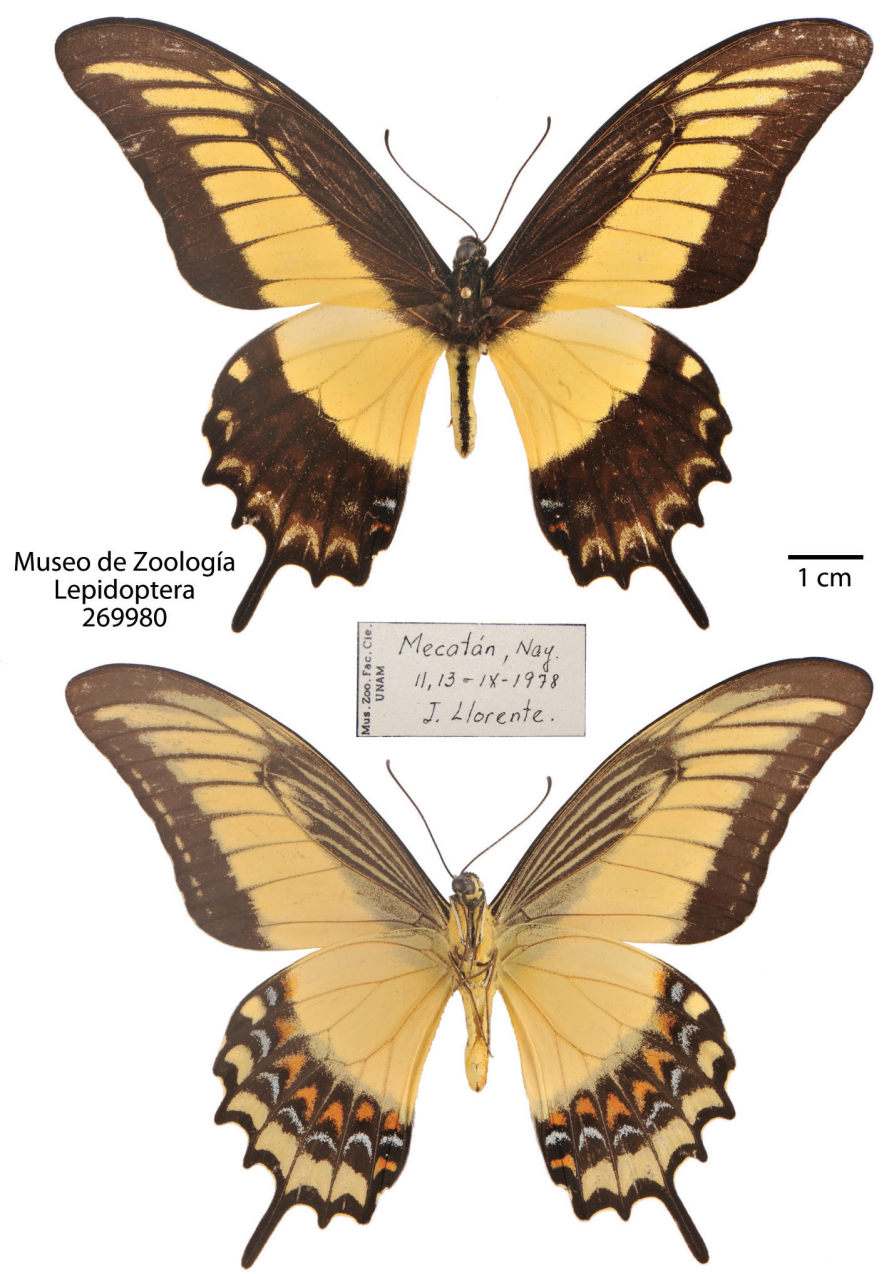

Fig. 2. Holotipo macho Heraclides androgeus reyesorum ssp. nov. México-Nayarit: Mecatán, 11-13/09/1978, J. Llorente leg., MZFC LEP-269980. Vistas dorsal (superior) y ventral (inferior).

Fig. 2. Holotype male Heraclides androgeus reyesorum ssp. nov. Mexico-Nayarit: Mecatán, 11-13/09/1978, J. Llorente leg., MZFC LEP-269980. Dorsal view (top) and ventral (bottom). 
manchas adyacentes, las cinco inferiores son trapezoidales, delimitadas por las venas negras; la primera subapical por lo general de longitud mayor, menos ancha que el resto, en ocasiones se encuentra dividida o reducida. La segunda y la tercera máculas son subcuadradas o elípticas alargadas. El margen exterior de las máculas 2-8 es variable, a veces convexo, en ocasiones cóncavo. Conforme se acercan al margen posterior las líneas en las venas son más estrechas.

Más allá del extremo apical de la $\mathrm{CD}$ y entre la costa, se presentan de una a tres manchas amarillas pequeñas, cada vez de menor amplitud hacia el margen costal, la del extremo apical es subtriangular, cuyo lado apical es cóncavo. Dentro de la $\mathrm{CD}$, entre $\mathrm{M}_{2} \mathrm{y} \mathrm{CuA}_{1}$, hay una mancha amarilla que puede ser doble, sencilla o ausente. La amplitud de la banda negra subterminal generalmente es de $1 / 2 \mathrm{a}^{2} / 3$ respecto a la amarilla medida en $\mathrm{CuA}_{1}$ (caracteres 3 y 4: Cuadro 1). Vista ventral: En su mayoría presenta un diseño muy similar al dorso, con amarillo más pálido $\left(\mathrm{N}_{00} \mathrm{~A}_{50} \mathrm{M}_{10}\right)$, que es más extenso en superficie. La CD en su mayor parte es marrón $\left(\mathrm{N}_{80} \mathrm{~A}_{30} \mathrm{M}_{30}\right)$, surcada longitudinalmente por cuatro líneas finas amarillo pálido $\left(\mathrm{N}_{20} \mathrm{~A}_{60} \mathrm{M}_{00}\right)$ con dos manchas distales hacia $\mathrm{M}_{1}-\mathrm{M}_{2}$ y $\mathrm{M}_{2}-\mathrm{M}_{3}$. La banda subterminal es más clara que el dorso; muy cercano al termen hay una fila de manchas amarillentas intercelulares, divididas en dos cada una, en general, desde las radiales hasta la anal.

Alas posteriores, vista dorsal: Base oscura reducida con bordes difusos. La banda amarilla es amplia, regularmente delimitada hacia el ápice de la $\mathrm{CD}$, a veces un poco postdiscalmente. A menudo las discocelulares $\mathrm{M}_{2}-\mathrm{M}_{3}$ (dci) y la $\mathrm{M}_{1}$ postdiscal son negras. En el ápice hay una mancha amarilla de forma y tamaño variado; en los espacios intervenales Rs- $\mathrm{CuA}_{2}$ se aprecian cerca del termen cinco lúnulas amplias amarillas, que son mayores al acercarse a la cubital. En el tornus casi siempre existe una mancha roja $\left(\mathrm{N}_{00} \mathrm{~A}_{90} \mathrm{M}_{90}\right)$ $\mathrm{y}$, superior a ésta, hay una más difusa azul pálido $\left(\mathrm{N}_{00} \mathrm{M}_{00} \mathrm{C}_{10}\right)$. En ocasiones, en el área postmediana se observan de una a cuatro manchas amarillas difusas.

El borde del termen es dentado por las prolongaciones ligeras en las venas y la cola en $\mathrm{M}_{3}$, que es de $15 \mathrm{~mm}$. Entre cada prolongación se exhiben cilia amarillo muy pálido. Vista ventral: De color amarillo pálido $\left(\mathrm{N}_{00} \mathrm{~A}_{30}\right.$ $\mathrm{M}_{00}$ ) desde la base hasta el área postmediana, poco más extendido que en vista dorsal. A continuación una franja marrón $\left(\mathrm{N}_{80} \mathrm{~A}_{80} \mathrm{M}_{60}\right)$, en ésta hay tres hileras sucesivas de manchas: la primera formada por siete sublúnulas en forma de punta de flecha de color naranja $\left(\mathrm{N}_{00}\right.$ $\mathrm{A}_{80} \mathrm{M}_{70}$ ), la del subápice es imperceptible; la segunda hilera, con siete lúnulas azul pálido $\left(\mathrm{N}_{00} \mathrm{M}_{00} \mathrm{C}_{10}\right)$, más estrechas y de límites más difusos; la última hilera es amarillo pálido, con cinco lúnulas muy amplias bien definidas 'molariformes' de gran tamaño y una apical subcuadrada. En el tornus se observa una mancha naranja $\left(\mathrm{N}_{00} \mathrm{~A}_{80} \mathrm{M}_{70}\right)$, que se aprecia en vista dorsal aunque más reducida. La lúnula situada en $\mathrm{M}_{3}-\mathrm{CuA}$ no se proyecta al inicio de la cola. Genitales: El proceso ventral del harpagón a todo lo largo bastante esclerosado; su primer tercio está abombado y su último tercio se dirige y acerca al proceso dorsal. Por tanto su margen interno es cóncavo. El proceso dorsal es subtriangular, cuya base es semejante a la altura; el margen interno se aprecia cóncavo; el margen dentado es heterogéneo. Edeago, su tamaño es similar a la longitud de la valva y es ensanchado en su base y recurvado en su parte media (Fig. 3).

\section{Hembra (Fig. 4) \\ Envergadura $\mathrm{AA}=63.31$ \\ (63-71mm, $n=16)$}

La cabeza, el tórax y el abdomen son de color negro por el dorso, con escamas amarillas en el borde interior de las tégulas. Vista ventral del tórax con dos franjas oblicuas amarillas; el abdomen con una franja de ese color a lo largo de las pleuras y debajo de éstas una más corta y angosta.

Alas anteriores, vista dorsal: Color de fondo negro verdoso $\left(\mathrm{N}_{90} \mathrm{C}_{80} \mathrm{~A}_{20}\right)$, claramente 


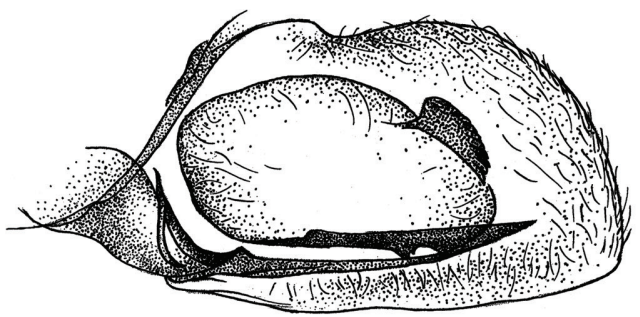

A
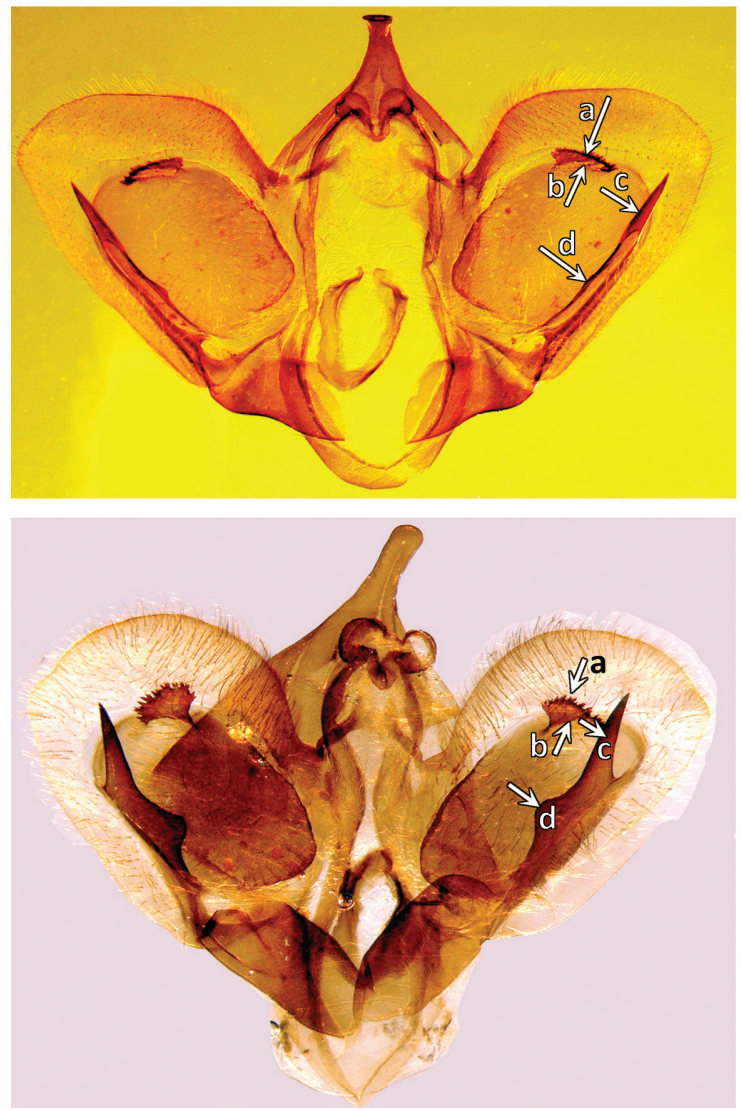
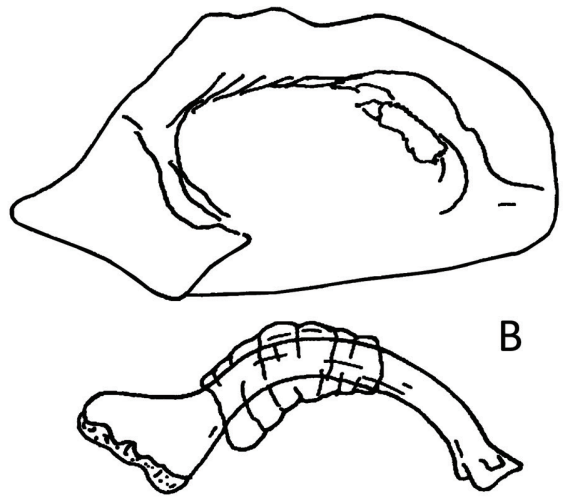

C

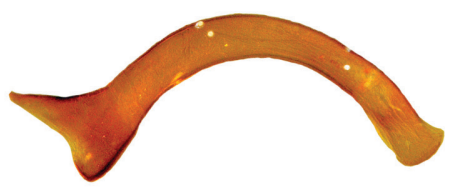

D

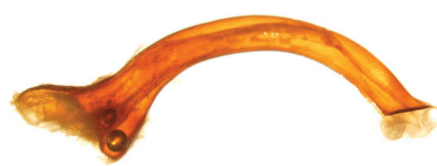

Fig. 3. Genitales masculinos de Heraclides androgeus. (A) Valva de H. a. epidaurus (redibujado de Godman, 1890: Biologia Centrali-Americana, Lámina 69); (B) Valva y edeago de H. a. epidaurus (esquema tomado de Beutelspacher \& Howe, 1984); (C) Valva y edeago de H. a. epidaurus (MHNCM-Museo de Historia Natural, Cd. México); (D) Valva y edeago (paratipo) de H. a. reyesorum ssp. nov. (holotipo); (a), (b) proceso esclerosado dorsal del harpagón; (c), (d) proceso esclerosado ventral del harpagón (fotografías de Blanca Claudia Hernández).

Fig. 3. Heraclides androgeus male genitals. (A) Valva of H. a. epidaurus (redrawn from Godman, 1890: Biologia CentraliAmericana, Plate 69); (B) Valva and aedeagus of H. a. epidaurus (diagram taken from Beutelspacher \& Howe, 1984); (C) Valva and aedeagus of H. a. epidaurus (MHNCM-Museum of Natural History, Mexico City); (D) Valva and aedeagus of H. a. reyesorum ssp. nov. (holotype); (a), (b) dorsal sclerotic process of harpe; (c), (d) ventral sclerotic process of harpe (pictures from Blanca Claudia Hernández). 


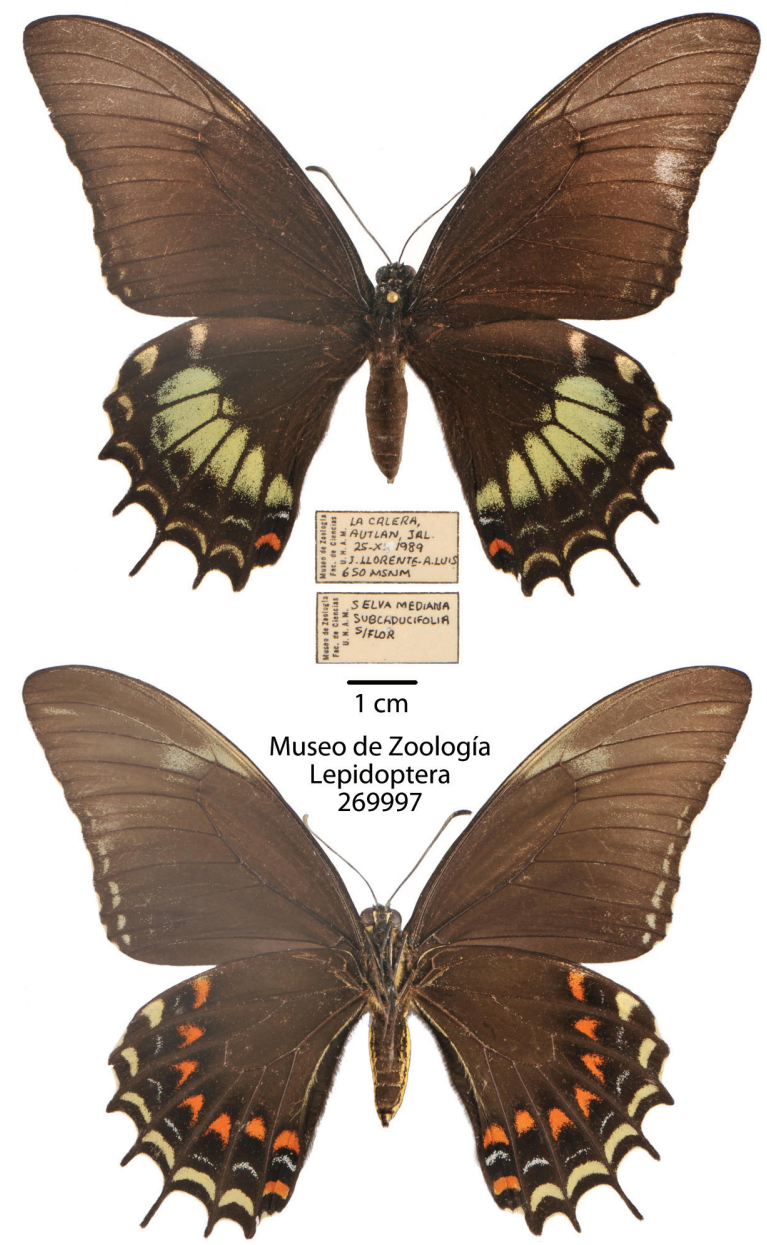

Fig. 4. Alotipo hembra Heraclides androgeus reyesorum ssp. nov. México-Jalisco: La Calera, Autlán, 25/10/1989, J. Llorente \& A. Luis leg., MZFC LEP-269997. Vistas dorsal (superior) y ventral (inferior).

Fig. 4. Allotype female Heraclides androgeus reyesorum ssp. nov. Mexico-Jalisco: La Calera, Autlán, 25/10/1989, J. Llorente \& A. Luis leg., MZFC LEP-269997. Dorsal view (top) and ventral (bottom).

despigmentadas a partir de la región postdiscal hasta la región apical y el área subterminal del tornus. En el área postdiscal-radial, hasta la costa, casi siempre se presentan manchas pequeñas amarillentas o vestigios de ellas. En la región subterminal del tornus a menudo se exhibe una fila de manchas amarillentas intercelulares pareadas. Ocasionalmente otra fila de tres manchas de escamas de color verde algo difusas, que convergen con la primera fila. Vista ventral: Color de fondo negro verdoso $\left(\mathrm{N}_{90} \mathrm{C}_{40} \mathrm{~A}_{60}\right)$ en la mitad basal hasta el área discal y el tornus; más claro en el resto del ala. Entre la zona apical de la CD con Sc, se presenta un área levemente amarillenta $\left(\mathrm{N}_{10} \mathrm{~A}_{30} \mathrm{M}_{00}\right)$, que alcanza hasta $\mathrm{M} 3$ en el área postmediana. En el área subterminal se aprecia una fila de cinco a seis manchas amarillentas sublunares, interrumpidas por el pliegue intercelular. En el termen se aprecian cilia desde el tornus, con límite en las radiales.

Alas posteriores, vista dorsal: Color de fondo negro verdoso $\left(\mathrm{N}_{90} \mathrm{C}_{80} \mathrm{~A}_{20}\right)$ mate, en la 
región subcostal es más claro; en el extremo de esta área hay dos manchas, la subapical amarillo o verde claro, y la apical amarillo claro brillante $\left(\mathrm{N}_{00} \mathrm{C}_{00} \mathrm{~A}_{60}\right)$. Postdiscalmente hasta más allá del área postmediana corre una banda que se reduce hacia el tornus en una serie de seis manchas verde claro $\left(\mathrm{N}_{00} \mathrm{C}_{20} \mathrm{~A}_{40}\right)$, delimitadas por las venas del color de fondo. En ocasiones en el ápice de la CD se presenta una manchita del mismo color. Más distalmente se forman tres marcas sublunares verde claro y una azul claro $\left(\mathrm{N}_{00} \mathrm{C}_{10} \mathrm{~A}_{10}\right)$ hacia el tornus. En la región subterminal hay cinco lúnulas de color verde claro y una mancha roja $\left(\mathrm{N}_{00}\right.$ $\mathrm{A}_{80} \mathrm{M}_{70}$ ) hacia el tornus. El margen externo es dentado con prolongaciones en las venas y una cola bien desarrollada en $\mathrm{M}_{3}$. El borde del margen presenta cilia intercelulares, a manera de seis sublúnulas estrechas blanquecinas. Vista ventral: Color de fondo negro verdoso $\left(\mathrm{N}_{90} \mathrm{C}_{80} \mathrm{~A}_{20}\right)$ mate, entre la base y ápice de $\mathrm{CD}$. En el área postmediana hasta el borde hay tres hileras de lúnulas sobre un fondo negro $\left(\mathrm{N}_{99} \mathrm{~A}_{99} \mathrm{C}_{99}\right)$, la primera hilera es postdiscal y formada por siete sublúnulas de color naranja $\left(\begin{array}{lll}\mathrm{N}_{00} & \mathrm{~A}_{80} & \mathrm{M}_{70}\end{array}\right)$; la segunda, son siete lúnulas muy estrechas azul pálido $\left(\mathrm{N}_{00} \mathrm{C}_{10} \mathrm{~A}_{00}\right)$ y la tercera en la región subterminal formada por seis lúnulas amarillo pálido $\left(\mathrm{N}_{10} \mathrm{~A}_{50} \mathrm{M}_{00}\right)$, bien definidas en todo el espacio intervenal y otra roja sobre el tornus.

Material examinado. Tipos: 62 ejemplares: 1 Holotipo, 1 Alotipo y 60 Paratipos, todos de México. Holotipo macho Nayarit: Mecatán, 11-13/09/1978, J. Llorente leg. MZFC LEP269980. Alotipo hembra: Jalisco, La Calera, Autlán, 25/10/1989, J. Llorente \& A. Luis leg., MZFC LEP-269997. Paratipos: NAYARIT: 6 ejemplares: El Izote, 1 ejemplar: 1 macho 24/06/1979, J. Llorente leg., MZFC LEP-269993; Laguna Santa María del Oro, 2 ejemplares: 1 macho 30/09/1996, A. Luis leg., MZFC LEP-32612, 1 hembra 30/09/1996, A. Luis leg., MZFC LEP-32616; Mecatán, 2 ejemplares: 1 macho 13/09/1978, A. Garcés M. leg., MZFC LEP-269990, 1 macho 13/09/1978, A. Garcés M. leg., MZFC LEP-269992; Singayta,
1 ejemplar: 1 macho 14/09/1978, J. Llorente leg., MZFC LEP-269991. JALISCO: 9 ejemplares: La Calera, 5 ejemplares: 1 hembra 15/11/1990, J. Llorente \& A. Luis leg., MZFC LEP-269995, 1 macho 05/06/1994, A. Luis leg., MZFC LEP-269977, 1 macho 05/06/1994, I. Vargas F. leg., MZFC LEP-269976, 1 macho 22/06/1991, I. Vargas F. leg., MZFC LEP269965, 1 macho 22/06/1991, I. Vargas F. leg., MZFC LEP-269968; Los Mazos, 2 ejemplares: 1 macho 12/11/1990, J. Llorente \& A. Luis leg., MZFC LEP-269967, 1 macho 26/09/1994, A. Luis \& J.L. Salinas leg., MZFC LEP-269996; Zenzontla, 2 ejemplares: 1 macho 08/06/1994, I. Vargas F. leg., MZFC LEP-269972, 1 macho 24/09/1994, A. Luis \& J.L. Salinas leg., MZFC LEP-269975. COLIMA: 11 ejemplares: Agua Dulce, 8 ejemplares: 1 macho 10/06/1994, A. Luis leg., MZFC LEP-269989, 1 macho 13/07/1995, A. Luis leg., MZFC LEP-269970, 1 macho 21/09/1989, J. Llorente \& A. Luis leg., MZFC LEP-269969, 1 macho 21/10/1989, J. Llorente \& A. Luis leg., MZFC LEP-269979, 1 macho 21/10/1989, J. Llorente \& A. Luis leg., MZFC LEP-269978, 1 macho 29/09/1989, J. Llorente \& A. Luis leg., MZFC LEP-269973, 1 macho 29/09/1989, J. Llorente \& A. Luis leg., MZFC LEP-269966, 1 macho 29/09/1989, J. Llorente \& A. Luis leg., MZFC LEP-269974; Agua Dulce-Platanarillo, 1 ejemplar: 1 macho 21/07/1989, A. Luis \& V. Bedoy leg., MZFC LEP-269971; Salada, la, 2 ejemplares: 1 macho 28/05/1983, L. González C. leg., MZFC LEP67633, 1 macho 28/06/1983, L. González C. leg., MZFC LEP-52482. MICHOACÁN: 14 ejemplares: Coahuayana, 1 ejemplar: 1 macho 8/1950, T. Escalante leg., MZFC LEP-53376; La Estanzuela, Chinicuila, 1 ejemplar: 1 hembra 15/09/1999, L. González C. leg., MZFC LEP-269998; La Nuez, 1 ejemplar: 1 hembra 09/08/1999, L. González C. leg., MZFC LEP269999; Rancho "El Zorrillo", Cañada Húmeda, 10 ejemplares: 1 hembra 02/09/1995, L. González C. leg., MZFC LEP-32617, 1 hembra 03/07/1995, L. González C. leg., MZFC LEP32621, 1 hembra 24/07/1995 MZFC LEP32614, 1 hembra 27/07/1995, L. González C. leg., MZFC LEP-32615, 1 macho 15/08/1995, 
L. González C. leg., MZFC LEP-32611, 1 hembra 18/08/1995, L. González C., MZFC LEP-32613, 1 hembra 25/08/1995, L. González C. leg., MZFC LEP-32620, 1 hembra 13/09/1995, L. González C. leg., MZFC LEP32619, 1 hembra 25/09/1995, L. González C. leg., MZFC LEP-32618, 1 macho s/f, L. González C. leg., MZFC LEP-32610; Santa Rosa, 1 ejemplar: 1 hembra 16/10/1991, L. González C. leg., MZFC LEP-269994. GUERRERO: 6 ejemplares: Acahuizotla, 4 ejemplares: 1 macho 01/09/1955 MZFC LEP-269981, 1 macho 01/10/1955, T. Escalante leg., MZFC LEP-269988, 1 macho 09/1955, T. Escalante leg., MZFC LEP-57456, 1 macho 09/1955, MZFC LEP-57457; Acapulco, 1 ejemplar: 1 macho 1975, T. Escalante leg., MZFC LEP57826; Tierra Colorada, Papagayo, 1 ejemplar: 1 hembra 1982, T. Escalante leg., MZFC LEP52696. OAXACA: 14 ejemplares: Azulillo 2 ejemplares: 1 macho 22/05/2008, O. Ávalos leg., MZFC LEP-269987, 1 macho 26/06/2008 MZFC LEP-269983; Candelaria Loxicha, 4 ejemplares: 1 macho 24/10/1990, J. Kemner leg., MZFC LEP-64855, 1 macho 7/1990, S.C. Lot leg., MZFC LEP-64860, 1 macho 8/1991, C. Almaraz leg., MZFC LEP-74760, 1 macho 8/1991, C. Almaraz leg., MZFC LEP-74759; Guichicovi-Zacatal-Tejón-Petapa, 2 ejemplares: 1 macho 05/06/2009, J. Kemner leg., MZFC LEP-269985, 1 macho 05/06/2009, J. Kemner leg., MZFC LEP-269986; cerca de Huatulco, 1 ejemplar: 1 macho 12/07/1991, J. Kemner leg., MZFC LEP-64854; La Pasionaria, 2 ejemplares: 1 macho 21/10/2008 MZFC LEP-269982, 1 macho 21/10/2008, J. Llorente \& A. Luis leg., MZFC LEP-269984; Zipolite, 1 ejemplar: 1 macho 24/07/1987, J. Kemner leg., MZFC LEP-64862; Santiago ChoapanLa Ermita, 2 ejemplares: 1 macho 20/04/1990 MZFC LEP- 64852, 1 macho 20/04/1990, J. Kemner leg., MZFC LEP- 64858.

Otro material consultado: Estos datos se utilizaron para fenología y distribución, 30 ejemplares. México. SONORA: 1 ejemplar: Santa Cruz 1/6/ (AME); SINALOA: 2 ejemplares: 1 ejemplar Villa Unión s/f USNM;
Villa Unión, 36.6 mi NE, 1 macho 02/07/1982 SDNHM; JALISCO: 4 ejemplares: Estación de Biología, UNAM, Chamela, 2 ejemplares: 2 machos 08/08/1975, CNIN LEP-10985, CNIN LEP-10988; Puerto Vallarta, 1 ejemplar: 1 macho 19/08/1951, CNIN LEP-10966; Tonila, 1 ejemplar: 1 macho 09/10/1982, SDNHM; COLIMA: 6 ejemplares: sin localidad, 1 ejemplar: 1 macho 1/10/1979, SDNHM; Los Mezcales, 1 ejemplar: 1 macho 16/09/1960, CNIN LEP-10965; La Salada, 3 machos 17/08/1975 CNIN LEP-10960, CNIN LEP-10992, CNIN LEP-10995, 1 macho 20/08/1982, SDNHM; MICHOACÁN: 1 ejemplar: Playa Azul, 1 macho 1/5/1975 CNIN LEP-10933; GUERRERO: 16 ejemplares: Mezcala, 4 ejemplares: 2 machos 1/7/1964 CNIN LEP-10935, CNIN LEP-10938; 2 machos 1/8/1964, CNIN LEP10928 CNIN LEP-10932; Playón, 3 ejemplares: 1 macho 1/9/1991 CNIN LEP-10943, 1 macho 1/8/1992 CNIN LEP-10941, 1 macho 1/10/1992 CNIN LEP-10942; Tierra Colorada, Papagayo, 2 ejemplares: 1 macho 1/8/1960, CNIN LEP-10934, 1 hembra, 1/8/1967, CNIN LEP-10944; Zihuatanejo, 7 ejemplares: 6 machos 1/7/1964 CNIN LEP-10929, CNIN LEP-10930, CNIN LEP-10931, CNIN LEP10936, CNIN LEP-10937, CNIN LEP-10940, 1 macho 1/8/1976 CNIN LEP-10939.

Etimología: El nombre subespecífico reyesorum está dedicado a los dos hijos de la primera autora: Margarita y Manuel Reyes.

Variabilidad y comparaciones: Las evidencias morfológicas muestran que reyesorum ssp. nov. es un taxón nuevo, pues se distingue de epidaurus por un conjunto de caracteres. Aunque en varios de ellos existe cierta variabilidad que se superpone parcialmente, algunos rasgos de los genitales son determinantes en cada subespecie.

En el cuadro 2 se analiza el conjunto de caracteres entre $H$. a. epidaurus y $H$. a. reyesorum ssp. nov. Primero se comparan los caracteres alares masculinos de H. a. reyesorum ssp. nov. у H. a. epidaurus, con relación a la envergadura y la amplitud de las bandas 
amarilla y negra en las AA. Luego se examinan los caracteres en AP, basados en el abdomen $\mathrm{y}$, finalmente, los caracteres genitales. Continúan caracteres femeninos y de distribución geográfica.

Los caracteres más significativos se cuantificaron en machos y se les asignó los números 1 a 4 y el carácter $3 / 4$ (Cuadro 1). En particular, en los caracteres 2 y 3 , se definieron tres amplitudes: banda angosta, mediana y ancha. Las diferencias fueron bastante claras cuando se compararon ordenadas y graficadas en diagramas de barras. El carácter 1 en reyesorum va de mediano a amplio y en epidaurus se ven todas las amplitudes; el carácter 2 y 3 siempre es angosto o mediano en reyesorum, en epidaurus comprende ejemplares con banda amarilla amplia (un tercio de la población). Respecto a la banda negra (carácter 4) que es un carácter complementario o relativo a los caracteres 2 y 3 (banda amarilla), se cumple que la mayor parte de los ejemplares de epidaurus es menor que en reyesorum, pero el tercio más amplio de epidaurus se superpone.

En machos de reyesorum la amplitud de las lúnulas amarillas situadas cerca del termen (Carácter 5, Cuadro 1) se aprecian de dos formas: amplias y medianamente amplias, las cuales en conjunto contrastan con las que presenta epidaurus, que en su inmensa mayoría son angostas. Un carácter equivalente a éste se presenta en hembras (Carácter 12, en Cuadro
1), el cual varía de la misma manera que en machos (Cuadro 2).

Al comparar los genitales en machos de las dos subespecies las diferencias son determinantes, lo que se expresa en el cuadro 2 y en la figura 3, en las que se presentan además, para propósitos de comparación, la valva y el edeago de $H$. androgeus epidaurus ilustrados en Godman \& Salvin (1890) (Fig. 3a) y Beutelspacher \& Howe (1984) (Fig. 3b) y las mismas estructuras genitales estudiadas por nosotros en H. androgeus epidaurus (Fig. 3c) y H.a. reyesorum ssp. nov. (Fig. 3d).

La presencia de formas ocres (Beutelspacher 1976) es muy excepcional y tal vez se deba a la oxidación de los papiliocromos expuestos a vapores y/o solventes en el caso de tales ejemplares expuestos a ello.

Prueba a caracteres cuantitativos de las AA de machos: Al comparar los cinco caracteres cuantitativos de AA (Cuadro 1), empleando la prueba U de Mann-Whitney se obtuvieron los resultados del cuadro 3. Éstos indican que todos los caracteres o variables comparados resultaron significativamente diferentes $(\mathrm{p}<0.01)$.

Distribución: $H$. androgeus reyesorum ssp. nov. se distribuye geográficamente en los estados costeros del Pacífico, de

CUADRO 3

Resultados de la prueba de U de Mann-Whitney aplicada a caracteres cuantitativos en alas anteriores, vista dorsal en machos

TABLE 3

Test results of Mann-Whitney U applied to quantitative characters in forewings, dorsal view in males

\begin{tabular}{ccccc} 
Variable-Carácter* & Media de H.a. epidaurus & Media de H.a. reyesorum ssp. nov. & Valor de U & Valor de $\mathrm{p}$ \\
1 & 60.20 & 62.19 & 1165.5 & 0.0009 \\
2 & 20.33 & 18.88 & 800.5 & $<0.001$ \\
3 & 13.75 & 13.04 & 1095.5 & 0.003 \\
4 & 5.95 & 7.31 & 433.5 & $<0.001$ \\
$3 / 4$ & 2.34 & 1.82 & 507.0 & $<0.001$ \\
\hline
\end{tabular}

Grados de libertad=115. Los caracteres alares indicados son: 1-Envergadura Alar; 2-Banda amarilla del margen posterior; 3-Banda amarilla de la vena $\mathrm{CuA}_{1} ; 4$-Banda negra de la vena $\mathrm{CuA}_{1} ; 3 / 4$-Cociente de caracteres 3 y 4. 
Sinaloa a Oaxaca (Fig. 5). El dato de Sonora debe confirmarse.

Hábitat: Esta subespecie se ha registrado en los estados, vegetaciones (se sigue a Rzedowski 1978) e intervalos de altitud (en metros sobre el nivel del mar) mostradas en el cuadro 4 y se resume como sigue: palmar de Attalea (20-150), bosque tropical subcaducifolio $=$ BTS (0-800), bosque de galería en bosque tropical caducifolio=BTC (0-800), bosque mesófilo de montaña=BMM (1030-1750) y bosque mixto de Quercus-Pinus=BPE (1160-1604). En la figura 6 se representa un perfil vegetacional de su hábitat en el estado de Nayarit, indicándose la localidad tipo.

El hábitat en el que se ha registrado su mayor abundancia se ubica en el bosque tropical caducifolio con bosque de galería, preferentemente entre los 500 y $800 \mathrm{~m}$ y en el intervalo inferior del bosque mesófilo de montaña, desde los 1000 a los $1750 \mathrm{~m}$.

Fenología: Las poblaciones exhiben protandria aparente, pues los machos aparecen más temprano que las hembras. La emergencia de machos es de abril a noviembre; en hembras de julio a noviembre. Ambos sexos alcanzan su mayor abundancia durante el mes de septiembre (Fig. 7), aunque los machos siempre son de tres a cuatro veces más abundantes en las muestras.

Los machos de $H$. androgeus epidaurus se presentan casi todo el año, aunque no se cuenta con registros de febrero. La mayor abundancia se alcanza durante los meses de junio a agosto. Las hembras se hallan de abril a octubre, alcanzando su mayor abundancia durante julio. Los fenómenos de protandria tal vez requieren una muestra mayor, aunque están muy extendidos en mariposas y muchos insectos (Nylin 2009: p. 212-213), pero en este caso se manifiesta con la muestra estudiada que para Papilionidae no es pequeña. No obstante, los datos también pueden indicar muestreo aún incompleto.

Endemismo: $H$. androgeus reyesorum ssp. nov. se encuentra en un área de endemismo sintetizada en el cuadro 5. En el apartado de diferenciación geográfica se discuten las comparaciones.

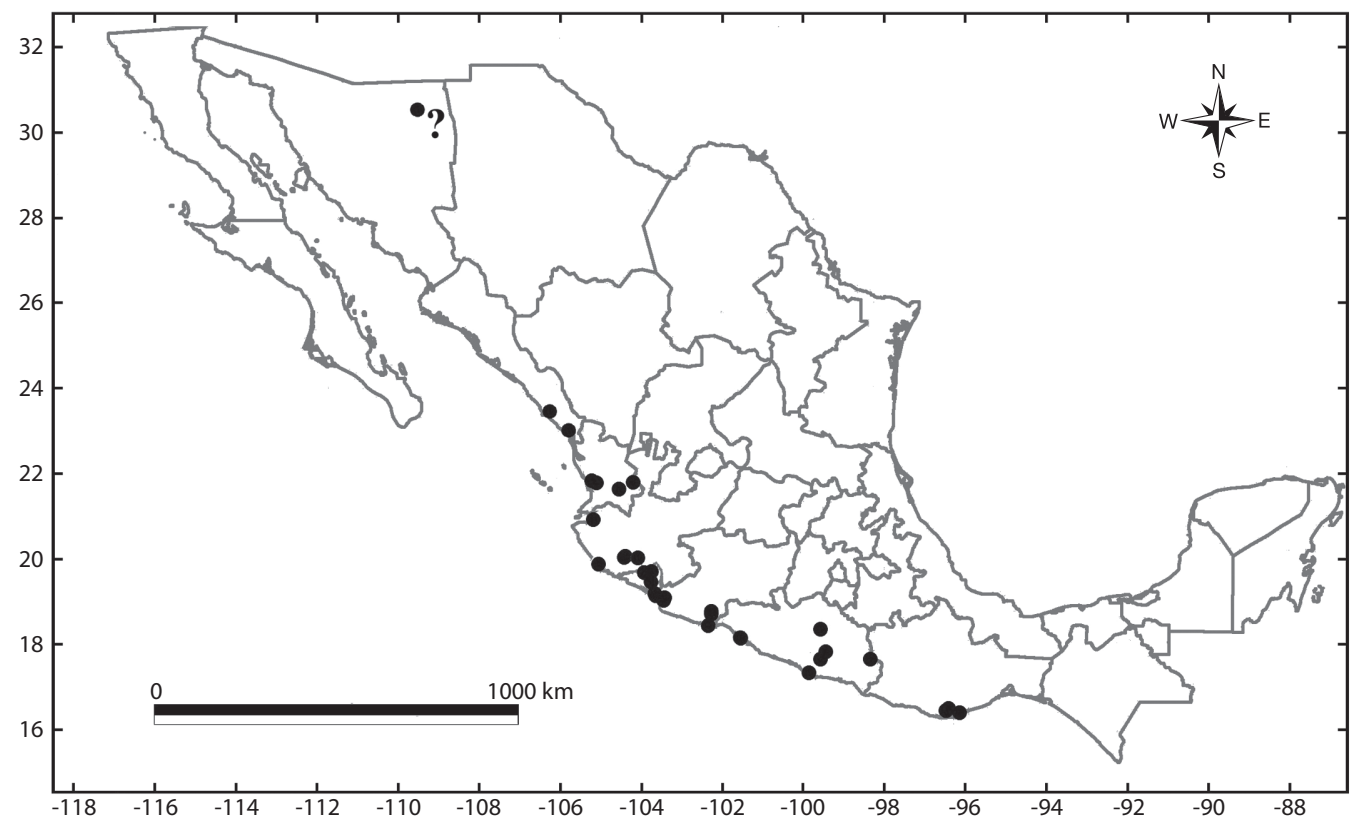

Fig. 5. Mapa de México. Distribución geográfica de H. a. reyesorum ssp. nov.

Fig. 5. Map of Mexico. Geographical distribution of H. a. reyesorum ssp. nov. 
TABLE 4

Heraclides androgeus reyesorum ssp. nov. hábitat

\section{CUADRO 4}

Hábitat de $H$. androgeus reyesorum ssp. nov.

\begin{tabular}{|c|c|c|}
\hline Estado & Tipo de vegetación* & Altitud (m) \\
\hline \multirow[t]{4}{*}{ Nayarit } & Palmar de Attalea & $20-150$ \\
\hline & BTC (bosque de galería) & $250-400$ \\
\hline & BMM & 1030 \\
\hline & BPE & 1160 \\
\hline \multirow[t]{5}{*}{ Jalisco } & BTC (bosque de galería) & 0 \\
\hline & BTS & 10 \\
\hline & BTS & 650 \\
\hline & BTC & 800 \\
\hline & BMM & $1600-1750$ \\
\hline \multirow[t]{2}{*}{ Colima } & BTS & 250 \\
\hline & BTC & 849 \\
\hline \multirow[t]{2}{*}{ Michoacán } & BTC & 37 \\
\hline & BPE & 1604 \\
\hline \multirow[t]{2}{*}{ Guerrero } & BTS & $0-800$ \\
\hline & BTC (bosque de galería) & $300-500$ \\
\hline \multirow[t]{3}{*}{ Oaxaca } & BTC & $30-217$ \\
\hline & BTS & 760 \\
\hline & BMM & $1500-1650$ \\
\hline
\end{tabular}

* Claves de vegetación utilizadas: BTC=bosque tropical caducifolio; $\mathrm{BMM}=$ bosque mesófilo de montaña; BPE=bosque mixto de Quercus-Pinus; BTS=bosque tropical subcaducifolio.

\section{DISCUSIÓN}

Comparación entre H. a. epidaurus y $H$. a. reyesorum ssp. nov.

Patrón alar: Los ejemplares de $H$. a. reyesorum ssp nov., a menudo son de mayor envergadura que en $H$. a. epidaurus. La amplitud de la banda amarilla en $H$. a. reyesorum ssp. nov. a menudo es más reducida que en H. a. epidaurus. La mayoría de los epidaurus muestran una banda negra más angosta que en los de reyesorum ssp. nov.

Las lúnulas amarillas en las AP de $H$. $a$. reyesorum ssp. nov. son de amplitud mayor.

En las AP de H. a. reyesorum ssp. nov. la banda negra postdiscal es más amplia y se acerca más a la $\mathrm{CD}$; en ocasiones la toca. Las venas discocelulares $\mathrm{M}_{2}-\mathrm{M}_{3}$ (dci) y $\mathrm{M}_{1}$ también son negras.

Las hembras de $H$. a. reyesorum ssp. nov. con frecuencia son de mayor envergadura que las de epidaurus.

El color verde de fondo de la región postmediana presenta algunas diferencias en AP; las lúnulas amarillas subterminales en vista ventral se presentan más reducidas en la mayoría de epidaurus y más anchas en la mayoría de H. a. reyesorum ssp. nov.

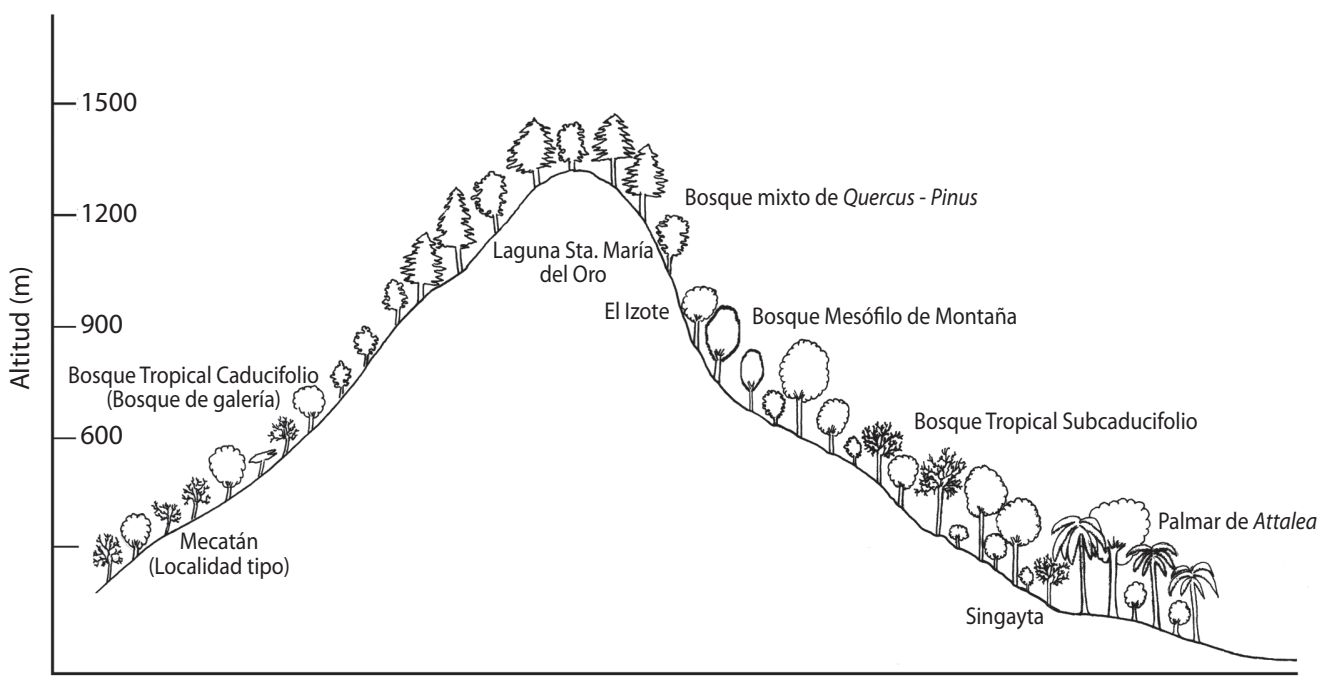

Fig. 6. Perfil vegetacional del hábitat de H. a. reyesorum ssp. nov. en el estado de Nayarit, incluyendo localidad tipo. Fig. 6. Vegetation profile from H. a. reyesorum ssp. nov. habitat from Nayarit state, including type locality. 


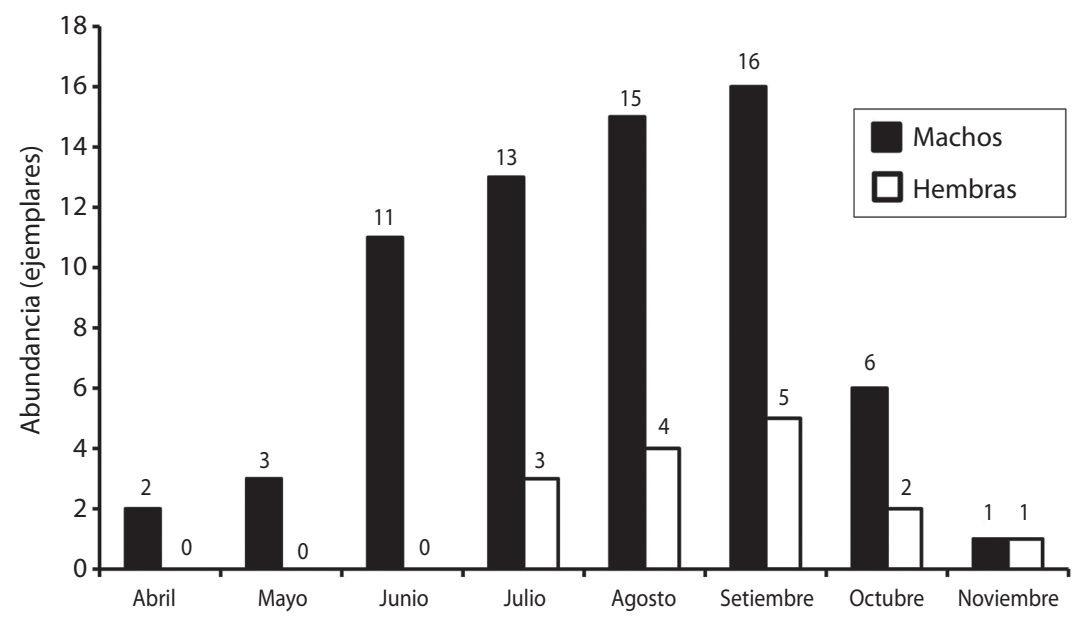

Fig. 7. Fenología de $H$. a. reyesorum ssp. nov.

Fig. 7. Phenology of $H$. a. reyesorum ssp. nov.

Abdomen: La banda negra pleural tiende a ser de mayor amplitud en $H$. androgeus reyesorum ssp. nov.

Genitales: La forma y las dimensiones de los dos procesos esclerosados del harpagón son distintos. En H. a. epidaurus el proceso dorsal es alargado en su base y los dientes más homogéneos; el de la parte ventral tiene forma de espina recta; mientras que en $H$. a. reyesorum ssp. nov. es una espina más gruesa y abombada en su primer tercio; su proceso dorsal se proyecta más y con los dientes más grandes y heterogéneos. El edeago es similar en dimensiones y curvatura en ambas subespecies.

Comparación con $H$. astyalus bajaensis (J.W. Brown \& Faulkner, 1992) y H. ornythion (Boisduval, 1836): Las convergencias o paralelismos con $H$. astyalus bajaensis se anotan a continuación:

1. En machos se observa en AA y AP la misma tendencia a la reducción de la banda postmediana de color amarillo $\mathrm{y}$, por consiguiente, la ampliación de la banda oscura.

2. La ampliación de las lúnulas amarillas subterminales en el dorso en AP, pero en vista ventral más amplias que en la dorsal.
3. En AA, en vista dorsal, conforme se acerca a la zona apical, las venas se aprecian más gruesas; las manchas que forman la banda están más separadas entre sí.

4. Amarillo más claro en vista ventral.

En Heraclides ornythion no ocurren estos paralelismos, pues en los machos la amplitud de la banda amarilla aumenta y las lúnulas son de mayor tamaño en la vertiente del Golfo y disminuyen en el Pacífico. Los ejemplares de ornythion, al parecer son extremadamente raros en el Pacífico.

Las lúnulas en AP, vista ventral, en ejemplares del Golfo prácticamente no existen, pues son una superficie continua de color amarillo, que se proyecta hacia la cola en $\mathrm{M}_{3}$, a diferencia de androgeus y astyalus, donde las colas son negras. En el Pacífico se observan como manchas definidas parecidas a las lúnulas, algunas subcuadradas, con un borde marginal más amplio que en el Golfo, aprox. 1.5 o 2 veces la amplitud con respecto al Pacífico.

Brown \& Faulkner (1984) comentaron acerca de la reducción de la banda amarilla en machos de $H$. astyalus de la vertiente del Pacífico, casi con apariencia de ornythion, pero de las hembras indican que parecen ser formas intermedias entre pallas y bajaensis o 
TABLE 5

Some papilionoids endemic to the Mexican Pacific Coast Province

\title{
CUADRO 5
}

Algunos papilionoideos endémicos de la Provincia Costa Pacífica Mexicana

\author{
Papilionidae \\ Protographium thyastes occidentalis (R.F. Maza, 1982) \\ Mimoides ilus occiduus (Vázquez, 1957) \\ Heraclides astyalus bajaensis (J.W. Brown \& Faulkner, 1992) \\ Priamides erostratus erostratinus (Vázquez, 1947) \\ Pterourus garamas baroni (Rothschild \& Jordan, 1906) \\ Pterourus menatius morelius (Rothschild \& Jordan, 1906)
}

\section{Pieridae}

Dismorphia amphione lupita Lamas, 1979

Dismorphia amphione isolda Llorente, 1984

Enantia mazai diazi Llorente, 1984

Lieinix neblina J. Maza \& R.G. Maza, 1984

Lieinix nemesis nayaritensis Llorente, 1984

Pereute charops leonilae Llorente, 1986

Pereute charops sphocra Draudt, 1931

Pereute charops nigricans Joicey \& Talbot, 1928

\section{Riodinidae}

Euselasia aurantiaca aurum Callaghan, Llorente \& Luis, 2007

Rhetus arcius beutelspacheri Llorente, 1988

Exoplisia azuleja Callaghan, Llorente \& Luis, 2007

Mesene jimena Callaghan, Llorente \& Luis, 2011

Lamphiotes velazquezi (Beutelspacher, 1976)

\author{
Nymphalidae \\ Opsiphanes blythekitzmillerae Austin \& A. Warren, 2007 \\ Consul electra adustus Lamas, 2003 \\ Archaeoprepona demophon occidentalis Stoffel \& Descimon, 1974 \\ Archaeoprepona demophoon mexicana Llorente, Descimon \& K. Johnson, 1993 \\ Catonephele cortesi R.G. Maza, 1982 \\ Eunica caelina agustina R.G. Maza, 1982 \\ Eunica malvina almae Vargas, Llorente \& Luis, 1996 \\ Diaethria anna mixteca J. Maza, 1977 \\ Myscelia cyaniris alvaradia R.G. Maza \& Díaz, 1982 \\ Diaethria asteria (Godman \& Salvin, 1894) \\ Actinote stratonice oaxaca (J.Y. Miller \& L.D. Miller, 1979) \\ Actinote guatemalena guerrerensis J. Maza, 1982 \\ Eueides isabella nigricornis R.G. Maza, 1982
}

las poblaciones del occidente entre Oaxaca y Sinaloa. Estos autores comentan que tienen una hembra atípica con una hilera de manchas rojonaranja bien desarrolladas en el dorso, como en pallas, y por las condiciones del ejemplar, no se aprecia la cola en $\mathrm{M}_{3}$.

Diferenciación geográfica: Numerosos casos en Papilionoidea se ajustan a la vicariancia biogeográfica entre $H$. a. epidaurus y $H$. a. reyesorum ssp. nov. Ambos taxones pertenecen a un patrón bien conocido. El aislamiento entre ambas vertientes de México, a nivel del Istmo de Tehuantepec como barrera fundamental, ha promovido diferenciación en muchos taxones, v. gr. Protographium thyastes, Heraclides astyalus, Pterourus garamas, Pterourus menatius, Enantia mazai, Lieinix 
nemesis, Dismorphia amphiona y Pereute charops como ejemplos de Papilionidae y Pieridae. Muchos casos más analizados reciente y sucintamente por Llorente \& Luis (1993, 1998), Luis et al. (2003), Oñate et al. (2006), Llorente et al. (2006), Luis et al. (2006) y Vargas et al. (2006) sintetizan este patrón biogeográfico.

Morrone \& Llorente (2006) describen este patrón de endemismo para Insecta, cuyos vicarios se presentan entre las provincias biogeográficas Costa Pacífica Mexicana y Golfo de México.

Los autores del presente trabajo han descrito endémicos de la Costa Pacífica Mexicana en las últimas tres décadas y cumplen con el patrón de vicariancia aludido. La historia de este patrón biogeográfico puede rastrearse a los trabajos clásicos de Godman \& Salvin (1878-1901) y Hoffmann (1940), pero después de ellos muchos autores han contribuido a su análisis con diversos trabajos taxonómicos en mariposas, v. gr. Vázquez $(1947,1957)$, L. \& J. Miller (1979), J. Maza et al. (1982), Brown \& Faulkner (1984), Llorente (1984, 1986, 1988) y R. Maza \& Turrent (1985).

Más recientemente, este patrón continúa consolidándose con los trabajos taxonómicos y de diferenciación geográfica publicados por Callaghan et al. $(2007,2011)$ en Riodinidae.

La gran mayoría de los vicarios que siguen este patrón se circunscriben al bosque tropical subcaducifolio y a la porción baja del bosque mesófilo de montaña, aunque los casos de diferenciación pueden ocurrir entre la porción superior del bosque mesófilo de montaña y el bosque mixto de Quercus-Pinus, estos casos pueden ser de grupos autóctonos diversificados en Mesoamérica (Halffter 1978), más que de filiación austral reciente. Además, tales casos pertenecen a las provincias Sierra Madre Oriental y del Sur, pues se registran a menudo en altitudes de 1500 a $2400 \mathrm{~m}$. Los endémicos estenoecos a bosque tropical caducifolio como Prestonia clarki y Ganyra kuschei podrían considerarse también en el mismo patrón de endemismo de $H$. a. reyesorum ssp. nov.

Dado que el patrón vicariante entre ambas vertientes comprende altitudes superiores, pero también el bosque tropical caducifolio, será necesario un análisis detallado e integral que incluya las diversas provincias biogeográficas, tipos de vegetación e intervalos altitudinales en los que las especies vicarias participan. En cierto modo Morrone \& Llorente (2006: 1020) lo expresan gráficamente.

Por lo pronto el caso de $H$. androgeus se ve apoyado biogeográficamente en su diferenciación por numerosos taxones.

Comentarios finales: Tyler et al. (1994) conformaron un grupo de especies en Heraclides comprendiendo $H$. ornythion, $H$. thersites (Fabricius, 1775), H. astyalus (Godart, 1819) y $H$. androgeus. En las cuatro especies el diseño alar y ambos procesos de los harpagones se asemejan y a la vez son distintos de otro grupo que comprende a $H$. paeon (Boisduval, 1836), H. cresphontes (Cramer, 1777), H. homothoas (Rothschild \& Jordan, 1906) y H. thoas (Linnaeus, 1771), cuyas similitudes en el diseño alar y los procesos de los harpagones también los acerca. Las especies de Heraclides de las Antillas son más divergentes y heterogéneas en caracteres alares y genitales pues parecen productos de barreras marítimas en el Caribe para estos taxones: H. aristodemus (Esper, 1794), H. aristor (Godart, 1819), H. caiguanabus (Poey, 1852), H. andraemon Hübner, 1823 y H. machaonides (Esper, 1796). Al examinar el patrón de los procesos de los harpagones entre las subespecies androgeus, epidaurus y reyesorum ssp. nov. se advierte gran semejanza entre ellas y a la vez diferencias más acentuadas con las especies de este grupo de Heraclides (Tyler et al. 1994: láms. 85-89). En estas similitudes y en las decisiones taxonómicas de Brown \& Faulkner (1984) y Lamas (2004) se inscribe nuestra consideración de aceptar a reyesorum como subespecie. No obstante, si se aplicase con generalidad a muchos Papilionidae los conceptos de especie evolutiva o filogenética tal vez podría elevarse a reyesorum ssp. nov. como una especie distinta y hermana de epidaurus. Hemos preferido ser conservadores hasta que un sistema de caracteres más 
amplio sea comprendido y analizado, incluyendo caracteres moleculares.

La variación paralela en el diseño de $H$. astyalus y $H$. a reyesorum ssp. nov., así como la concentración de subespecies de papiliónidos en la costa pacífica mexicana citada por Tyler et al. (1994), Llorente \& Luis (1993, 1998) y Oñate et al. (2006) nos apoya en nuestra decisión sistemática.

\section{AGRADECIMIENTOS}

Agradecemos a las siguientes personas e instituciones su invaluable ayuda en el trabajo: A Gerardo Lamas (Lima, Perú) por proporcionarnos la foto digital del holotipo macho de $H$. androgeus epidaurus. A las siguientes personas de la Facultad de Ciencias: Omar Ávalos por el tratamiento estadístico y su apoyo en la toma de fotografías, a Adrián Emilio Flores por los dibujos de genitales masculinos de Biologia Centrali-Americana, a José Luis Salinas por la producción del mapa, a Claudia Hernández Mejía por la disección y toma de fotografías de los genitales masculinos de $H$. androgeus reyesorum ssp. nov. y a Susana Valencia (Herbario FCME) por su ayuda con la nomenclatura de los tipos vegetacionales. A Ma. Eugenia Díaz Batres del MHNCM por facilitarnos un ejemplar macho de $H$. androgeus epidaurus y una laminilla de los genitales del mismo. A Martha Montemayor y Paula López (Centro de Estudios Clásicos, UNAM) y Abner Chiquieri (Brasil) por su ayuda en la traducción de la descripción original en latín de H. a. epidaurus. A la Facultad de Ciencias y a los proyectos PAPIME, PAPIIT IN 203509 y 214212, CONACyT 083237 y 177292, y UC MEXUS-CONACyT los cuales han financiado muchos viajes de campo, que sirvieron para obtener los ejemplares, base de este estudio. Los comentarios de Olaf H.H. Mielke fueron de gran valor en el manuscrito final.

\section{RESUMEN}

Hemos reunido evidencia de que Heraclides está formado por dos subespecies, cada una con caracteres propios que las distinguen; analizamos, describimos y nominamos a Heraclides androgeus reyesorum ssp. nov. que se distribuye en la costa pacífica mexicana. Los principales caracteres masculinos fueron la amplitud de las bandas amarilla y negra en alas anteriores, que al cuantificarse y analizarse tuvieron una diferencia significativa entre las poblaciones de las dos vertientes de México, a pesar que algunos caracteres son variables y muestran superposición parcial. En alas posteriores las diferencias fueron la amplitud de lúnulas subterminales en vista dorsal y ventral. Se analizaron los genitales masculinos, se encontraron diferencias determinantes en los procesos esclerosados del harpagón. Las diferencias subespecíficas entre hembras se refieren al brillo y extensión de las manchas verdes en alas posteriores y a la amplitud de lúnulas en vista ventral. La mayor abundancia de $H$. a. reyesorum ssp. nov. fue en el bosque de galería del bosque tropical caducifolio y en el intervalo inferior del bosque mesófilo, a 500-800m y 1 000-1 750m, respectivamente. Se discute la existencia de un patrón de endemismo analizado por Halffter (1978) y Morrone \& Llorente (2006) que obedece a procesos históricos vicariantes y resume la presencia de subespecies nuevas en el Pacífico como en $H$. androgeus y muchos otros taxones.

Palabras clave: Papilionidae, endemismo, vicariancia, distribución, costa pacífica Mexicana, bosque tropical subcaducifolio, genitales.

\section{REFERENCIAS}

Beutelspacher, C.R. 1976. Nuevas formas de papiliónidos mexicanos. Rev. Soc. Mex. Lep. 2: 61-70.

Beutelspacher, C.R. \& W.H. Howe. 1984. Mariposas de México I. Introducción y generalidades. Superfamilia Papilionoidea. La Prensa Médica Mexicana, D.F., México.

Brown, J.W. \& D.K. Faulkner. 1984. Distributional records of certain Rhopalocera in Baja California, Mexico, with the description of a new subspecies of Papilio (Heraclides) astyalus (Godart) (Lepidoptera: Papilionidae). Bull. Allyn Mus. 83: 1-9.

Callaghan, C.J., J. Llorente-B. \& A.M. Luis. 2007. Descriptions of four new Mexican riodinids. (Lepidoptera: Riodinidae). Zootaxa 1660: 33-43.

Callaghan, C.J., J. Llorente-B. \& A.M. Luis. 2011. A new mexican Mesene (Lepidoptera: Riodinidae). Zootaxa 2896: 53-64.

Godman, F.D. \& O. Salvin. 1878-1901. Biologia Centrali-Americana. Insecta. Lepidoptera-Rhopalocera 2: 209-240. Dulau \& Co., Bernard Quaritch, Londres, Inglaterra.

Halffter, G. 1978. Un nuevo patrón de dispersión de la Zona de Transición Mexicana: El Mesoamericano de Montaña. Fol. Entomol. Mex. 39-40: 219-222.

Hoffmann, C.C. 1940. Catálogo Sistemático y Zoogeográfico de los lepidópteros mexicanos. Primera Parte. 
Papilionoidea. An. Inst. Biol. Univ. Nac. Autón. Méx., Ser. Zool. 11: 639-739.

Howe, W.H. 1975. The Butterflies of North America. Doubleday \& Company, Inc. Nueva York, EEUU.

Klüppers, H. 1996. Atlas de los colores. Blume, Barcelona, España.

Lamas, G. (ed.). 2004. Checklist: part 4B Hesperioidea Papilionoidea. In J.B. Heppner. Atlas of Neotropical Lepidoptera 5B. Association for Tropical Lepidoptera, Florida, EEUU.

LeCrom, J.F., J. Llorente-B., L.M. Constantino \& J.A. Salazar. 2004. Mariposas de Colombia. Vol. 2. Pieridae. Carlec, Bogotá, Colombia.

Llorente-B., J. 1984. Sinopsis sistemática y biogeográfica de los Dismorphiinae de México con especial referencia del género Enantia Huebner (Lepidoptera: Pieridae). Fol. Entomol. Mex. 58: 1-207.

Llorente-B., J. 1986. Las razas geográficas de Pereute charops (Boisduval, 1836) con la descripción de una nueva subespecie. An. Inst. Biol. Univ. Nac. Autón. Méx., Ser. Zool. 56: 245-258.

Llorente-B., J. 1988. Las poblaciones de Rhetus arcius en México con notas sobre las subespecies sudamericanas. (Lepidoptera: Lycaenidae, Riodinidae). An. Inst. Biol. Univ. Nac. Autón. Méx., Ser. Zool. 58: 241-258.

Llorente-B., J. \& A.M. Luis. 1993. A conservation oriented analysis of mexican butterflies: the Papilionidae (Lepidoptera: Papilionoidea), p. 147-177. In T.P. Ramammorthy, J. Fa, R. Bye \& A. Lot (eds.). The biological diversity of Mexico: origins and distributions. Oxford University.

Llorente-B., J. \& A.M. Luis. 1998. Análisis conservacionista de las mariposas mexicanas: Papilionidae (Lepidoptera: Papilionoidea), p. 149-178. In $\mathrm{T}$. Ramammorthy, R. Bye, A. Lot \& J. Fa (eds.). Diversidad Biológica de México: orígenes y distribución. Instituto de Biología, UNAM, México.

Llorente-B., J., A.M. Luis, I.F. Vargas \& A.D. Warren. 2004. Butterflies of the state of Nayarit, Mexico. J. Lepid. Soc. 58: 203-222.

Llorente-B., J., M.O. Trujano, A.M. Luis, J. Castro \& I.F. Vargas. 2006. Patrones de distribución de la familia Pieridae (Lepidoptera) de México, p. 715-770. In J.J. Morrone \& J. Llorente-B. (eds.). Componentes bióticos principales de la entomofauna mexicana, Vol. II. Las Prensas de Ciencias, UNAM, D.F., México.

Luis, A.M., J. Llorente-B., I.F. Vargas \& A.D. Warren. 2003. Biodiversity and Biogeography of mexican butterflies
(Lepidoptera: Papilionoidea and Hesperioidea). Proc. Entomol. Soc. Wash. 105: 209-224.

Luis, A.M., M.O. Trujano, J. Llorente-B. \& I.F. Vargas. 2006. Patrones de distribución de la subfamilias Danainae, Apaturinae, Biblidinae y Heliconiinae (Lepidoptera: Nymphalidae), p. 771-865. In J.J. Morrone \& J. Llorente-B. (eds.). Componentes bióticos principales de la entomofauna mexicana, Vol. II. Las Prensas de Ciencias, UNAM, D.F., México.

Maza, J., R.G. Maza \& R.F. Maza. 1982. Lepidópteros nuevos del Estado de Guerrero, México. (Papilionoidea). Rev. Soc. Mex. Lep. 7: 2-14.

Maza, R.G. \& R. Turrent. 1985. Mexican Lepidoptera. Eurytelinae I. Publicaciones especiales. Soc. Mex. Lep. 4. D.F., México.

Miller, J.Y. \& L.D. Miller. 1979. Notes on Mexican Actinote (Nymphalidae: Acraeinae) and their relatives, with description of a new subspecies. J. Lepid. Soc. 32: 261-272.

Morrone, J.J. 2001. Biogeografía de América Latina y el Caribe. M \& T-Manuales \& Tesis SEA, vol. 3. Zaragoza, España.

Morrone, J.J. \& J. Llorente-B. 2006. Conclusiones. In J.J. Morrone \& J. Llorente-B. (eds.). Componentes bióticos principales de la entomofauna mexicana, Vol. II. Las Prensas de Ciencias, UNAM, D.F., México.

Nylin, S. 2009. Gradients in butterfly biology, p. 198-216. In J. Settele, T. Shreeve, M. Konvicka \& H. Van Dyck (eds.). Ecology of butterflies in Europe. Cambridge University, Cambridge, Reino Unido.

Oñate, L.O., M.O. Trujano, J. Llorente-B., A.M. Luis \& I.F. Vargas. 2006. Patrones de distribución de la familia Papilionidae (Lepidoptera), p. 661-714. In J.J. Morrone \& J. Llorente-B. (eds.). Componentes bióticos principales de la entomofauna mexicana, Vol. II. Las Prensas de Ciencias, UNAM, D.F., México.

Rothschild, L.W. \& H.E. Jordan. 1906. A revision of the American Papilios. Novit. Zool. 13: 411-752.

Rzedowski, J. 1978. Vegetación de México. Limusa. D.F., México.

Tyler, H.A., K.S. Brown Jr. \& K.H. Wilson. 1994. Swallowtail butterflies of the Americas: a study in biological dynamics, ecological diversity, biosystematics, and conservation. Scientific Publishers, Gainesville, Florida, EEUU.

Vargas, I.F., J. Llorente-B. \& A.M. Luis. 1999. Distribución de los Papilionoidea (Lepidoptera: Rhopalocera) de la Sierra de Manantlán (250-1650 m.s.n.m.) en los estados de Jalisco y Colima. Publ. Espec. Mus. Zool., UNAM, Fac. Ciencias, UNAM 11: 1-153. 
Vargas, I.F., A.M. Luis, J. Llorente-B. \& A.D. Warren. 1996. Butterflies of the state of Jalisco, Mexico. J. Lepid. Soc. 50: 97-138.

Vargas, I.F., M.O. Trujano, J. Llorente-B. \& A.M. Luis. 2006. Patrones de distribución de las subfamilias Ithomiinae, Morphinae y Charaxinae (Lepidoptera: Nymphalidae), p. 867-943. In J.J. Morrone \& J. Llorente-B. (eds.). Componentes bióticos principales de la entomofauna mexicana, Vol. II. Las Prensas de Ciencias, UNAM, D.F., México.
Vázquez, L. 1947. Papilios nuevos de México. An. Inst. Biol. Univ. Nac. Autón. Méx., Ser. Zool. 18: 249-256.

Vázquez, L. 1957. Papilios nuevos de México. IV. An. Inst. Biol. Univ. Nac. Autón. Méx., Ser. Zool. 27: 473-485.

Warren, A.D. \& J. Llorente-B. 1999. Butterflies of Mismaloya, Jalisco and adjacent parts of Banderas Bay and Southern Nayarit, México. Dugesiana 6: 17-32.

Warren, A.D., I.F. Vargas, A.M. Luis \& J. Llorente-B. 1998. Butterflies of the state of Colima, Mexico. J. Lepid. Soc. 52: 40-72.

\section{APÉNDICE 1 \\ Material examinado y consultado de Heraclides androgeus epidaurus Los acrónimos de las colecciones aparecen en Material y Métodos. $\mathrm{s} / \mathrm{f}=$ ejemplares sin fecha disponible \\ APPENDIX 1 \\ Material examined and consulted of Heraclides androgeus epidaurus}

NUEVO LEÓN: 1 ejemplar: Entre Montemorelos y Rayones, 1 macho 22/11/1986, MZFC LEP-32604; TAMAULIPAS: 1 ejemplar: Matamoros, 1 ejemplar s/f, AMNH; SAN LUIS POTOSÍ: 5 ejemplares: Cañón Palitla, Tamazunchale, 2 ejemplares: 1 macho 01/09/1978, LACM, 1 macho 07/06/1979, LACM; Crucero Xilitla, 1 ejemplar: 1 macho 09/07/1983, LACM; La Herradura, 1 ejemplar s/f, AMNH; cerca de Xilitla, 1 ejemplar: 1 macho 07/07/1991, MZFC LEP sin número; HIDALGO: 1 ejemplar: Puerto del Caballo, 1 macho 10/09/1978, LACM; PUEBLA: 19 ejemplares: La Ceiba, 2 ejemplares: 1 macho 13/04/1979, MZFC LEP-59682, 1 hembra 20/05/1979, MZFC LEP-59683; Barranca de Patla, 4 ejemplares: 1 macho 21/08/1977, MZFC LEP-59681, 2 machos 1/7/1978, CNIN LEP-10978, CNIN LEP-10990, 1 hembra 20/07/1979, CNIN LEP-11009; Puebla, 1 ejemplar s/f, AMNH; Tequezquitla, 11 ejemplares: 1 macho 17/10/1976, MZFC LEP59684, 1 macho 08/04/1979, MZFC LEP-59685, 1 hembra 17/08/1979 MZFC LEP-59687, 3 machos 02/09/1979, MZFC LEP-59689, MZFC LEP-67746, MZFC LEP59688, 1 macho 04/09/1979, MZFC LEP-59686, 1 hembra 1/8/1972, CNIN LEP-11007, 1 macho 04/07/1981, CNIN LEP-10982, 1 hembra 1/7/1983, CNIN LEP-11008, 1 macho 1/7/1983, CNIN LEP-10989; Villa Juárez, 1 ejemplar: 1 hembra s/f, MZFC LEP-44399; OAXACA: 54 ejemplares: Cerro Armadillo, 4 ejemplares: 4 machos 11/07/1981, MZFC LEP-59674, MZFC LEP-59675, MZFC LEP-59673, MZFC LEP-67634; La Esperanza, 1 ejemplar: 1 macho 11/03/1980, MZFC LEP-59677; La Gringa, Santa María Chimalapa, 2 ejemplares: 2 machos 02/10/1995 MZFC LEP-55238, MZFC LEP-55237; La Soledad, 1 ejemplar: 1 hembra 21/07/1979 MZFC LEP59678; Matías Romero, 1 ejemplar: 1 hembra 1/9/1965 CNIN LEP-11004; Metates, 3 ejemplares: 1 hembra 23/08/1976 CNIN LEP-11010, 1 macho 28/03/1984 CNIN LEP-10991, 1 macho 1/7/1991 CNIN LEP-10984; Metates, 13 ejemplares: 1 hembra 22/07/1979 MZFC LEP-59679, 1 macho 08/06/1982 MZFC LEP-57675, 1 macho 07/1991 MZFC LEP-64859, 1 hembra 05/1992 MZFC LEP-59671, 9 machos 06/1992 MZFC LEP-32602, MZFC LEP-32596, MZFC LEP-32597, MZFC LEP-32598, MZFC LEP74755, MZFC LEP-32609, MZFC LEP-32605, MZFC LEP-32603, MZFC LEP-32592; Naranjal Chiltepec, 5 ejemplares: 1 macho 03/1981, MZFC LEP-44392; 1 hembra 19/04/1982, MZFC LEP-67635, 1 hembra 20/06/1986, MZFC LEP-57719, 1 hembra 01/08/1980, MZFC LEP59676, 1 macho 11/07/1981, MZFC LEP-44393; Rancho Sarabia, 1 ejemplar: 1/9/ , AME; San José Chiltepec, 5 ejemplares: 1 macho 1/10/1962, CNIN LEP-10973, 1 hembra 1/7/1963, CNIN LEP-11005, 1 hembra 08/07/1973, SDNHM, 1 ejemplar 08/07/1973, SDNHM, 1 hembra 10/07/1973, SDNHM; San Martín Soyolapan, 1 ejemplar: 1 macho 01/10/1982, MZFC LEP-57686; San Mateo Yetla, $3 \mathrm{~km}$ S Valle Nacional, 1 ejemplar: 1 macho 12/05/1988, MZFC LEP-57664; Sierra de Juárez, 7 ejemplares: 3 machos 06/1992, MZFC LEP-32593, MZFC LEP-64853, MZFC LEP-64857, 4 machos 08/1992, MZFC LEP74845, MZFC LEP-32594, MZFC LEP-32601, MZFC LEP-74846; Soyolapan El Bajo, 7 ejemplares: 1 hembra 01/10/1982, MZFC LEP-44398, 1 macho 1/1/1963, CNIN LEP-10969, 2 machos 1/8/1963, CNIN LEP-10974, CNIN LEP-10977, 1 hembra 1/6/1964, CNIN LEP-11001, 1 hembra 28/07/1964, CNIN LEP-11006, 1 ejemplar s/f, AMNH; Tuxtepec, 1 ejemplar 1/9/, AME; Usila, Tuxtepec, 1 ejemplar, 1 macho s/f, CNIN LEP-10968; VERACRUZ: 77 ejemplares: Barranca de Cayoapa, Tejería, 2 ejemplares: 1 hembra 29/07/1978, MZFC LEP-44404, 1 hembra 12/10/1980, MZFC LEP-44403; Catemaco, 2 ejemplares: 1 macho 16/09/1958, CNIN LEP-10946, 1 ejemplar 1/6/, AME; Cerro El Vigía, Santiago Tuxtla, 23 ejemplares: 1 macho 18/08/1963, MZFC LEP-44396, 1 hembra 30/07/1982, MZFC LEP-44406, 1 hembra 15/07/1983, MZFC LEP-67636, 1 hembra 16/09/1985, MZFC LEP44407, 1 hembra 10/09/1987, MZFC LEP-67637, 1 macho 
1/3/1963, CNIN LEP-10955, 1 macho 01/06/1963, CNIN LEP-10964, 1 hembra 02/08/1963, CNIN LEP-10997, 4 machos 02/08/1963, CNIN LEP-10958, CNIN LEP10959, CNIN LEP-10962, CNIN LEP-10963, 1 macho 20/09/1963, CNIN LEP-10961, 1 macho 1/9/1963, CNIN LEP-10956, 1 macho 1/10/1963, CNIN LEP-10950, 3 machos 1/3/1964, CNIN LEP-10948, CNIN LEP-10949, CNIN LEP-10952, 1 hembra 03/06/1964, CNIN LEP10998, 4 machos, 1/9/1964, CNIN LEP-10951, CNIN LEP-10953, CNIN LEP-10954, CNIN LEP-10957; Córdoba, 4 ejemplares: 2 ejemplares 1/5/1916, LACM, 2 ejemplares s/f, USNM; Cuetzalapan, 5 ejemplares: 1 macho 03/05/1981, CNIN LEP-10993, 2 hembras 1/9/1981, CNIN LEP-11002, CNIN LEP-11003, 2 machos 1/9/1981, CNIN LEP-10986, CNIN LEP-10987; Dos Amates, Catemaco, 2 ejemplares: 1 hembra 1/8/1962, CNIN LEP-11000, 1 ejemplar 1/8/, AME; El Trapiche, 3 km S de Teocelo, 1 ejemplar: 1 hembra 23/08/1980, MZFC LEP-44405; Hacienda "El Mirador", 1 ejemplar s/f, AMNH; Misantla, 1 ejemplar s/f, AMNH; Motzorongo, 6 ejemplares: 2 ejemplares 1/10/1906, USNM, 2 ejemplares 1/6/, USNM, 1 ejemplar 1/8/, AME, 1 ejemplar s/f, AMNH; Orizaba, 1 ejemplar s/f, AMNH; Poblado de Cárdenas, Los Tuxtlas, 1 ejemplar: 1 hembra 17/04/1974, MZFC LEP-44410; Potrero Viejo, Paraje Nuevo, 1 ejemplar: 1 hembra 02/05/1953, CNIN LEP-10999; Presidio, Ixhuatlán del Café, 12 ejemplares: 1 macho 07/1951, MZFC LEP-44397, 2 machos 1/10/1939, SDNHM, 1 macho 1/7/1940, CNIN LEP-10945, 2 ejemplares 1/6/1941, USNM, 2 hembras 1/5/, SDNHM, 2 machos 1/5/, SDNHM, 1 ejemplar 1/8/, AME, 1 ejemplar s/f, AMNH; Pureza, 1 ejemplar: 1 macho 1/1/1935, CNIN LEP-10967; Santiago Tuxtla, 5 ejemplares: 1 hembra 8/1970, MZFC LEP-44400, 2 hembras 09/1976, MZFC LEP-44401, MZFC LEP-44402, 2 machos 09/1976, MZFC LEP-44394, MZFC LEP-44395; Tapalapan, 3 ejemplares: 1 macho 04/10/1980, MZFC LEP-67748, 1 hembra 20/07/1982, MZFC LEP-44408, 1 hembra 25/08/1982, MZFC LEP-44409; Tezonapa Huapacal, 2 ejemplares: 2 machos 1/6/1946, CAS, sin localidad, 1 ejemplar: 1 macho 1/7/1960, CNIN LEP-10981; Volcán Santa Marta, 1 ejemplar: 1 macho 1/8/1987, CNIN LEP-11887; Xalapa, 3 ejemplares s/f, AMNH; Zapoapan de Cabañas, 1 ejemplar: 1 macho 29/12/1954, CNIN LEP-10947; TABASCO: 1 ejemplar: Tepescuintle, 1 ejemplar s/f, AMNH; CHIAPAS: 42 ejemplares: Arriaga, 1 ejemplar: 1 hembra s/f, CNIN LEP-10996; Bonampak, \pm 10 mi NW, 1 ejemplar: 1 macho 30/07/1988, MZFC LEP-64861; Bonampak, $11.5 \mathrm{mi}$ al $\mathrm{N}$ de San Javier, 9 ejemplares: 1 macho 25/05/1979, MZFC LEP-44391, 3 machos 06/05/1988, MZFC LEP-32595, MZFC LEP-32599, MZFC LEP32606, 3 machos 08/05/1988, MZFC LEP-32607, MZFC LEP-74756, MZFC LEP-32600, 1 macho 02/05/1978, CNIN LEP-10983, 1 macho 23/05/1984, CNIN LEP10994; Campamento arqueológico del INAH de Yaxchilán, 7 ejemplares: 1 macho 21/06/1998, MZFC LEP-36783, 3 machos 21/08/1998, MZFC LEP-40012, MZFC LEP40013, MZFC LEP-40014, 1 macho 23/08/1998, MZFC LEP-40010, 1 macho 26/08/1998, MZFC LEP-39993; sin localidad, 1 ejemplar, 1/7/, AME; Curva NE de la Omega, Monumento Natural Yaxchilán, 7 ejemplares: 2 machos 21/06/1998, MZFC LEP-36789, MZFC LEP-36790, 5 machos 25/06/1998, MZFC LEP-36785, MZFC LEP36787, MZFC LEP-36788, MZFC LEP-36786, MZFC LEP-36784; Mazatán, 1 ejemplar: 1/7/, AME; Monumento Natural Yaxchilán, Porción N de la Omega, 1 ejemplar: 1 macho 22/08/1998, MZFC LEP-39992; Palenque, 2 ejemplares: 1 macho 20/07/1975, MZFC LEP-59672, 1 ejemplar 1/6/, AME; Palenque-Ocosingo, km 95, s/carretera, 2 ejemplares: 2 machos 16/07/1991, MZFC LEP-32608, MZFC LEP-64856; San Antonio Buenavista (Santa Rosa), Independencia, 1 ejemplar: 1 hembra 10/1982, MZFC LEP-67632; Santa Rosa, Comitán, 1 ejemplar: 1/6/ , AME; Yaxchilán, 7 ejemplares: 4 machos 1/4/1964, CNIN LEP10970, CNIN LEP-10971, CNIN LEP-10975, CNIN LEP10976, 1 macho 1/8/1964, CNIN LEP-10980, 1 macho 1/6/1966, CNIN LEP-10972, 1 macho 1/7/1966, CNIN LEP-10979; Zona arqueológica de Yaxchilán, 3 ejemplares: 2 machos 23/08/1998, MZFC LEP-40011, MZFC LEP-39994, 1 ejemplar 1/6/, AME; QUINTANA ROO: 1 ejemplar: Nuevo X-Can, 1 macho 26/05/1982 (MZFC).

\section{APÉNDICE 2}

Medidas de caracteres alares en machos utilizadas para prueba U de Mann-Whitney. Las claves de caracteres corresponden a: 1-Envergadura Alar; 2-Banda amarilla del margen posterior; 3-Banda amarilla de la vena CuA ; 4-Banda negra de la vena $\mathrm{CuA}_{1} ; 3 / 4$-Cociente de caracteres 3 y 4

\section{APPENDIX 2}

Measurements of wing characters in males used in Mann-Whitney U Test. The character codes correspond to: 1-Wing Span; 2 - Yellow band of posterior margin; 3-Yellow band of vein $\mathrm{CuA}_{1}$; 4-Black band of vein $\mathrm{CuA}_{1}$; $3 / 4$-Ratio of characters 3 and 4

H. androgeus epidaurus (Machos, Alas Anteriores)

\begin{tabular}{cccccccc} 
Ejemplar & Número Colección & 1 & 2 & 3 & 4 & Carácter $3 / 4$ \\
1 & 44395 & 61.17 & 20.27 & 13.18 & 7.04 & 1.87 \\
2 & 44394 & 53.31 & 16.3 & 11.68 & 5.85 & 2.00 \\
3 & 67748 & 60.52 & 20.56 & 14.82 & 6.44 & 2.30 \\
4 & 44396 & 64.89 & 20.14 & 14.19 & 7.3 & 1.94 \\
\hline
\end{tabular}




\begin{tabular}{|c|c|c|c|c|c|c|}
\hline Ejemplar & Número Colección & 1 & 2 & 3 & 4 & Carácter $3 / 4$ \\
\hline 5 & 44397 & 49.2 & 14.61 & 10.37 & 5.72 & 1.81 \\
\hline 6 & 59684 & 65 & 22.52 & 15.24 & 6.58 & 2.32 \\
\hline 7 & 59685 & 67.42 & 24.97 & 16.79 & 5.6 & 3.00 \\
\hline 8 & 59688 & 63.56 & 21.39 & 15.04 & 6.35 & 2.37 \\
\hline 9 & 32604 & 55.9 & 19.17 & 12.46 & 5.23 & 2.38 \\
\hline 10 & 59686 & 62.3 & 20.69 & 14.53 & 5.52 & 2.63 \\
\hline 11 & 59681 & 52.29 & 16.92 & 11.8 & 6.73 & 1.75 \\
\hline 12 & 59689 & 55.8 & 17.76 & 11.41 & 5.24 & 2.18 \\
\hline 13 & 59682 & 63.35 & 21.11 & 15.66 & 6.11 & 2.56 \\
\hline 14 & 59680 & 59.86 & 19.45 & 13.17 & 7.76 & 1.70 \\
\hline 15 & 67746 & 58.13 & 20.91 & 14.36 & 5.14 & 2.79 \\
\hline 16 & 64856 & 59.02 & 19.52 & 14.08 & 5.54 & 2.54 \\
\hline 17 & 39993 & 62.85 & 19.49 & 14.08 & 6.65 & 2.12 \\
\hline 18 & 36784 & 54.09 & 19.62 & 12.04 & 4.99 & 2.41 \\
\hline 19 & 36789 & 60.28 & 20.02 & 14.8 & 5.33 & 2.78 \\
\hline 20 & 32600 & 62.76 & 21.76 & 14.74 & 5.86 & 2.52 \\
\hline 21 & 32595 & 61.76 & 21.47 & 13.4 & 6.39 & 2.10 \\
\hline 22 & 44391 & 60.79 & 19.96 & 12.81 & 7 & 1.83 \\
\hline 23 & 32606 & 62.92 & 22.02 & 14.14 & 5.62 & 2.52 \\
\hline 24 & 32599 & 66.38 & 22.36 & 14.12 & 7.21 & 1.96 \\
\hline 25 & 36790 & 58.88 & 18.73 & 11.98 & 6.63 & 1.81 \\
\hline 26 & 59672 & 62.7 & 22.51 & 16.08 & 5.91 & 2.72 \\
\hline 27 & 36788 & 55.7 & 19.04 & 11.26 & 6.55 & 1.72 \\
\hline 28 & 36786 & 61.3 & 20.95 & 13.19 & 5.77 & 2.29 \\
\hline 29 & 74756 & 59.17 & 22.42 & 13.57 & 5.44 & 2.49 \\
\hline 30 & 40014 & 62.17 & 20.1 & 14.34 & 5.27 & 2.72 \\
\hline 31 & 32607 & 65.21 & 24.71 & 16.36 & 6.01 & 2.72 \\
\hline 32 & 36785 & 53.09 & 16.73 & 10.39 & 5.67 & 1.83 \\
\hline 33 & 36787 & 58.01 & 20.01 & 13.2 & 5.33 & 2.48 \\
\hline 34 & 36783 & 54.92 & 19.1 & 11.03 & 6.58 & 1.68 \\
\hline 35 & 64861 & 62.29 & 19.73 & 13.83 & 7.2 & 1.92 \\
\hline 36 & 39992 & 59.81 & 21.4 & 14.79 & 5.6 & 2.64 \\
\hline 37 & 32608 & 65.2 & 23.65 & 15.19 & 5.38 & 2.82 \\
\hline 38 & 39994 & 61.13 & 20.22 & 14.44 & 5.67 & 2.55 \\
\hline 39 & 40010 & 56.13 & 16.72 & 12.09 & 5.73 & 2.11 \\
\hline 40 & 40012 & 59.54 & 19.49 & 13.77 & 5.92 & 2.33 \\
\hline 41 & 40011 & 59.33 & 20.29 & 11.98 & 7.12 & 1.68 \\
\hline 42 & 40013 & 61.96 & 21.43 & 14.23 & 6.37 & 2.23 \\
\hline 43 & 57675 & 57.77 & 21.32 & 14.33 & 4.88 & 2.94 \\
\hline 44 & 44392 & 62.9 & 23.18 & 15.81 & 5.35 & 2.96 \\
\hline 45 & 55238 & 61.38 & 20.65 & 14.26 & 5.75 & 2.48 \\
\hline 46 & 55237 & 62.42 & 20.48 & 14.41 & 6.01 & 2.40 \\
\hline 47 & 59673 & 60.88 & 20.11 & 15.16 & 5.21 & 2.91 \\
\hline 48 & 67634 & 49.81 & 17.15 & 11.42 & 4.34 & 2.63 \\
\hline 49 & 59677 & 62.5 & 21.57 & 15.05 & 4.76 & 3.16 \\
\hline 50 & 59675 & 55.21 & 18.26 & 11.53 & 6.41 & 1.80 \\
\hline 51 & 57686 & 56.43 & 19.66 & 11.42 & 5.04 & 2.27 \\
\hline 52 & 57664 & 60.96 & 22.07 & 14.28 & 4.7 & 3.04 \\
\hline 53 & 44393 & 57.13 & 18.29 & 12.92 & 5.9 & 2.19 \\
\hline 54 & 32603 & 65.44 & 23.23 & 15.49 & 5.64 & 2.75 \\
\hline 55 & 64859 & 60.95 & 20.51 & 14.44 & 5.47 & 2.64 \\
\hline 56 & 32609 & 63.32 & 20.26 & 15.07 & 6.57 & 2.29 \\
\hline 57 & 74845 & 59.22 & 19.62 & 11.34 & 6.67 & 1.70 \\
\hline 58 & 32605 & 62.47 & 21.92 & 14.75 & 6.36 & 2.32 \\
\hline 59 & 59674 & 59.64 & 20.29 & 13 & 6.49 & 2.00 \\
\hline 60 & 74846 & 59.96 & 19.47 & 13.92 & 5.63 & 2.47 \\
\hline 61 & 32596 & 59.83 & 20.15 & 13.84 & 5.39 & 2.57 \\
\hline 62 & 32592 & 56.96 & 18.79 & 13.31 & 5.23 & 2.54 \\
\hline 63 & 32593 & 64.15 & 22.39 & 15.65 & 5.92 & 2.64 \\
\hline
\end{tabular}




$\begin{array}{cccccccc}\text { Ejemplar } & \text { Número Colección } & 1 & 2 & 3 & 4 & \text { Carácter } 3 / 4 \\ 64 & 64853 & 64.87 & 22.23 & 15.64 & 5.71 & 2.74 \\ 65 & 74755 & 61.13 & 18.97 & 13.19 & 7.66 & 1.72 \\ 66 & 32601 & 63.84 & 19.33 & 13.2 & 6.82 & 1.94 \\ 67 & 32602 & 61.8 & 20.65 & 15.35 & 5.58 & 2.75 \\ 68 & 32594 & 61.31 & 21.05 & 13.87 & 5.57 & 2.49 \\ 69 & 64857 & 60.75 & 21.34 & 13.9 & 6.76 & 2.06 \\ 70 & 32598 & 59.95 & 19.83 & 14.91 & 5.79 & 2.58 \\ 71 & 32597 & 61.54 & 20.72 & 14.57 & 5.79 & 2.52\end{array}$

H. androgeus reyesorum ssp. nov. (Machos, Alas Anteriores)

\begin{tabular}{|c|c|c|c|c|c|c|}
\hline Ejemplar & Número Colección & 1 & 2 & 3 & 4 & Carácter $3 / 4$ \\
\hline 1 & 269993 & 60.89 & 18.66 & 13.56 & 7.74 & 1.752 \\
\hline 2 & 32612 & 59.27 & 18.23 & 11.77 & 6.53 & 1.802 \\
\hline 3 & 269992 & 61.34 & 14.65 & 10.82 & 9.25 & 1.170 \\
\hline 4 & 269980 & 60.74 & 16.3 & 12.29 & 6.62 & 1.856 \\
\hline 5 & 269990 & 60.32 & 15.64 & 11.11 & 7.07 & 1.571 \\
\hline 6 & 269991 & 63 & 18.38 & 13.44 & 6.93 & 1.939 \\
\hline 7 & 52482 & 60.8 & 19.92 & 13.95 & 6.37 & 2.190 \\
\hline 8 & 67633 & 60.47 & 20.07 & 12.72 & 6.36 & 2.000 \\
\hline 9 & 269989 & 65.6 & 19.66 & 13.09 & 8.95 & 1.463 \\
\hline 10 & 269969 & 62.75 & 19.49 & 12.58 & 7.89 & 1.594 \\
\hline 11 & 269970 & 59.89 & 16.79 & 11.49 & 7.23 & 1.589 \\
\hline 12 & 269968 & 63.95 & 19.86 & 12.98 & 7.94 & 1.635 \\
\hline 13 & 269966 & 66.95 & 17.18 & 12.5 & 8.42 & 1.485 \\
\hline 14 & 269965 & 67.1 & 19.5 & 12.9 & 8.56 & 1.507 \\
\hline 15 & 269974 & 63.28 & 19.39 & 13.26 & 7.81 & 1.698 \\
\hline 16 & 269973 & 64.85 & 18.12 & 13 & 8.17 & 1.591 \\
\hline 17 & 269972 & 65.2 & 21.08 & 14.33 & 7.67 & 1.868 \\
\hline 18 & 269971 & 60.76 & 18.8 & 12.84 & 7.43 & 1.728 \\
\hline 19 & 269967 & 67.68 & 19.39 & 13.96 & 9.25 & 1.509 \\
\hline 20 & 269979 & 66.08 & 18.92 & 14.55 & 7.19 & 2.024 \\
\hline 21 & 269978 & 67.86 & 19.47 & 14.67 & 7.64 & 1.920 \\
\hline 22 & 269977 & 64.59 & 20.54 & 13.38 & 7.7 & 1.738 \\
\hline 23 & 269976 & 62.37 & 19.77 & 12.77 & 8.04 & 1.588 \\
\hline 24 & 269975 & 68.23 & 22.36 & 15.14 & 7.99 & 1.895 \\
\hline 25 & 64855 & 51.28 & 17.8 & 10.34 & 6.21 & 1.665 \\
\hline 26 & 269987 & 61.45 & 18.52 & 13.34 & 7.74 & 1.724 \\
\hline 27 & 269986 & 60.23 & 19.1 & 13.18 & 7.08 & 1.862 \\
\hline 28 & 269983 & 61.01 & 18.69 & 12.71 & 6.38 & 1.992 \\
\hline 29 & 74759 & 60.1 & 19.02 & 13.2 & 6.09 & 2.167 \\
\hline 30 & 64862 & 61.96 & 18.11 & 12.71 & 7.39 & 1.720 \\
\hline 31 & 64854 & 58.81 & 17.93 & 11.92 & 6.94 & 1.718 \\
\hline 32 & 269982 & 57.58 & 19.82 & 14.83 & 6.1 & 2.431 \\
\hline 33 & 269985 & 57.33 & 18 & 12.85 & 5.76 & 2.231 \\
\hline 34 & 269984 & 62.56 & 19.86 & 13.68 & 7.41 & 1.846 \\
\hline 35 & 64860 & 63.44 & 20.03 & 14.27 & 7.08 & 2.016 \\
\hline 36 & 74760 & 58.04 & 19.29 & 11.13 & 7.3 & 1.525 \\
\hline 37 & 64852 & 62.46 & 22.31 & 14.79 & 5.58 & 2.651 \\
\hline 38 & 64858 & 65.44 & 22.34 & 15.66 & 5.62 & 2.786 \\
\hline 39 & 57456 & 65.75 & 18.88 & 13.19 & 8.29 & 1.591 \\
\hline 40 & 57457 & 60.51 & 17.53 & 13.32 & 6.29 & 2.118 \\
\hline 41 & 57826 & 57.56 & 17.43 & 11.72 & 6.4 & 1.831 \\
\hline 42 & 269981 & 61.39 & 19.99 & 13.95 & 6.6 & 2.114 \\
\hline 43 & 269988 & 61.51 & 18.49 & 12.98 & 7.16 & 1.813 \\
\hline 44 & 53376 & 61.28 & 18.06 & 11.84 & 8.98 & 1.318 \\
\hline 45 & 32611 & 64.03 & 18.61 & 12.94 & 7.38 & 1.753 \\
\hline 46 & 32610 & 63.17 & 16.68 & 12.1 & 7.63 & 1.586 \\
\hline
\end{tabular}


